\title{
QUATRIÈME PARTIE
}

\section{Nécessité de recourir à des modélisations et à des observations}

"Comple tenu de la complexité même du système (Planète Terre), la compréhension de son évolution et la quantification de son état actuel nécessitent une double approche fondée d'une part sur la collecte des données, d'autre part sur la modélisation ». 

Les différentes contributions des parties deux et trois, qui traitent d'écosystèmes ou de compartiments physiques, permettant d'aborder la question des cycles d'un point de vue systémique, mettent clairement en avant le besoin de données et de modèles. Certains textes soulignent que la complexité de ces systèmes se traduit par une difficulté de construction de modèles jamais monodisciplinaires et mentionnent aussi les difficultés d'obtention des données (métrologie, observations de moyens et long termes, stratégie d'échantillonnage). On peut souligner que, comme dans toutes les sciences expérimentales, l'observation joue un rôle essentiel sans que, le plus souvent, son articulation avec la modélisation conceptuelle ou la simulation numérique soit bien appréhendée ${ }^{1}$. Cette articulation est pourtant le point fondamental, qui nous amène d'ailleurs à traiter la question des observatoires et de la modélisation dans la même partie de cet ouvrage. En effet, si l'on suit la formalisation de Tarantola (1987), reprise par Courtier (2003), au-delà de la seule mesure, une observation est un triptyque constitué par une représentation du système étudié - un modèle - l'observation au sens commun - la mesure - et un opérateur d'observation qui permet de relier l'espace modèle et l'espace des mesures. Le problème « direct » est résolu par l'opérateur d'observation et permet de confronter la modélisation aux observations. Symétriquement, le problème « inverse » permet, connaissant les observations, d'inférer les paramètres essentiels du modèle. II ne s'agit donc plus d'opposer l'expérimentation/observation à la modélisation, mais bel et bien de mieux faire collaborer ces deux méthodologies, qui ont chacune leurs difficultés propres.

\section{Les modèles}

L'espace modèle est, pour la plupart des questions relatives aux cycles biogéochimiques, constitué de plusieurs champs quadridimensionnels ou tridimensionnels. En effet les phénomènes étudiés sont instationnaires et non homogènes spatialement dès lors que l'on sort d'expérimentations en conditions contrôlées, ce qui est le cas de l'étude in situ sur les écosystèmes cités dans les chapitres précédents. La modélisation impose alors de commencer par identifier les échelles de variabilité qui sont significatives, ainsi que les variables pertinentes pour chaque question et donc chaque champ considéré. Cette étape permet notamment de réduire les dimensions d'étude et de modélisation (milieux stratifiés, par exemple). On utilise en fait une connaissance a priori des milieux considérés. Cette question préalable à la construction de modèles est notamment l'objet du chapitre 9, qui, outre une typologie sommaire des modèles, développe la question des grandeurs caractéristiques, échelles d'observation, maillage et approximation, ainsi que le délicat problème du changement d'échelles et des

\footnotetext{
${ }^{1} C^{\prime}$ est un constat qui a déjà été effectué dans le RST no 15 : Études sur l'environnement : de l'échelle du territoire à celle du continent, juin 2003.
} 
couplages. On notera que ces questions sont tout à fait sous-jacentes dans les chapitres de la première partie, où les échelles, notamment temporelles, associées aux éléments peuvent être très différentes.

La question de l'approximation et du maillage, et donc de la discrétisation du modèle pour obtenir une simulation numérique, met en lumière la collaboration avec les données d'observation. L'utilisation de l'assimilation de données est certainement la meilleure illustration de cette collaboration données-modèle, au bénéfice du modèle et aussi des données. L'utilisation de ces techniques est maintenant opérationnelle depuis plusieurs décennies pour la simulation atmosphérique. Elles sont cependant toujours en cours de développement pour la chimie, la biosphère et l'hydrologie.

\section{Données et observatoires}

Les données nécessaires à la connaissance des cycles biogéochimiques proviennent d'observations issues de disciplines différentes éventuellement gérées par des autorités différentes. Ces deux remarques mettent en évidence des difficultés dans les collaborations nécessaires entre chercheurs, sur le plan métrologique, sur les protocoles d'échanges de données, sur la politique de gestion des données, pour ' 'archivage sur le long terme. Le rapport sur les observatoires pour l'environnement (ORE et $O O E$ ) remis aux ministères en charge de l'Environnement et en charge de la Recherche en 2001 développe cette analyse.

La pérennisation des observations est une clé pour les domaines traités ici. En effet, les échelles de temps en jeu sont longues, même si certains phénomènes ont, eux, une durée caractéristique beaucoup plus courte. Les différents réseaux d'observatoires décrits dans cette partie illustrent cette nécessité du long terme. Il faut remarquer que cette pérennisation ne relève pas seulement d'une approche volontariste. Elle doit être assortie d'une analyse approfondie de la rationalité des réseaux et des possibilités de collaboration.

À titre d'exemple, on peut citer le cas du cycle du carbone : les agences spatiales ont été amenées à élaborer des missions observant la couleur de l'eau et donc des planctons. On a pu noter un manque de coordination inter-agences dans les années 1980 et 1990 avec des périodes comprenant plusieurs missions simultanées et d'autres sans observations. Malgré la mise en place d'un réseau de surveillance de la pollution atmosphérique (Bapmon), on manque aujourd'hui d'observations du dioxyde et monoxyde de carbone et du méthane à l'échelle globale. 
Dans ce qui suit, des observatoires spécifiques pour la biogéochimie sont présentés avec leurs propres échelles de temps et d'espace.

Le compartiment océanique tient une place toute particulière à l'échelle du globe : par son volume, par l'importance des flux de matière et d'énergie qu'il induit, il conditionne en grande partie l'environnement terrestre. Les observatoires y sont relativement anciens, probablement du fait que les difficultés techniques inhérentes au milieu ont forcé les scientifiques à faire converger les moyens dans une coordination internationale. La biogéochimie des océans s'aborde à plusieurs échelles spatiotemporelles : la question de l'impact des gaz à effet de serre sur le climat mondial et du rôle régulateur de l'océan est désormais l'un des grands enjeux de l'océanographie. L'échelle de travail est a priori océanique, même si la contribution de la zone côtière léchelle régionale, en interaction avec l'échelle globale) reste difficile à aborder et mal connue. En revanche, l'impact des pollutions d'origine anthropique, le plus souvent côtières bien que parfois hauturières en cas de marée noire, s'aborde à l'échelle locale, voire régionale. Les observatoires de long terme permettent d'enregistrer les pollutions accidentelles et de les situer dans un contexte temporel. Néanmoins, dans le contexte actuel d'éradication progressive de ces événements, ils constituent surtout l'unique moyen de mise en évidence des évolutions lentes dues aux pollutions chroniques et diffuses. L'identification et la maîtrise de ces contaminations constitue le réel enjeu des années à venir. La modélisation constitue l'outil intégrateur de ces différentes problématiques, qui ne sont pas totalement étanches.

Le bilan des écosystèmes forestiers est complexe à évaluer ; ceux-ci constituent néanmoins un compartiment biogéochimique incontournable. La dynamique humaine peut y être dramatiquement rapide (déforestation, pluies acides, incendies...), comparée aux échelles de temps naturelles qui sont lentes. L'observation à long terme est donc là aussi cruciale et s'entend à échelle régionale, voire globale. La modélisation permet non seulement de rendre compte de ces différentes dynamiques, mais aussi de transformer les paramètres directement observables (densité des plantations, qualité, nature des sols...) en informations intégrées, comme les flux de dioxyde de carbone.

La problématique des radionucléides est plus spécifique, mais intimement liée aux réseaux de suivi et d'observation. Organisés dans un triple objectif politique, sanitaire et scientifique, ces observatoires ont beaucoup apporté aux sciences de l'environnement, à toutes les échelles : globale en ce qui concerne les trajectoires atmosphériques, la dynamique des océans... mais aussi très locale lorsque l'on aborde la stratification sédimentaire et les différents cycles dans le sol. Ces mesures de traceurs très spécifiques sont «idéales 》 en matière de développement, calage et validation de modèles. 



\section{Développements de la modélisation - Nécessité de mise en place $d^{\prime}$ indicateurs}

CLAUDINE SCHMDT-LANE ET MARE-HELENE TUSSEAU-VULLEMN 



\section{Introduction - État et tendance actuels de la modélisation des cycles biogéochimiques}

La biogéochimie, dont le principal objet d'étude concerne les bilans, les transferts et les cycles d'éléments naturels ( $C, N, P, S$, etc.), nécessite la prise en compte de processus qui relèvent à la fois de la biologie, de la chimie et de la physique. Cette nécessaire imbrication des disciplines est à l'origine d'un certain nombre de difficultés liées notamment à la grande disparité entre les formalismes chimiques, physiques et biologiques ${ }^{1}$, ce qui rend difficile leur développement interactif.

L'étude des cycles biogéochimiques repose alors sur l'obtention de données et leur nécessaire intégration dans des modèles issus d'assemblages plus ou moins complets, de modèles bio/géo/chimiques intégrant des processus physiques. L'une des difficultés de cet assemblage réside dans la différence de maturité des modèles de ces divers champs disciplinaires.

Par exemple, les modèles de la chimie reposent sur les principes thermodynamiques et permettent, moyennant une bonne connaissance analytique du système, $d$ 'en prévoir de manière déterministe et fiable l'état d'équilibre, mais aussi l'évolution cinétique. On pourrait imaginer traiter de la même façon certains niveaux d'organisation biologique, dans la mesure où la biochimie a permis de décrire et de modéliser certains mécanismes complexes (par exemple, la photosynthèse, le cycle de Krebs, etc. ${ }^{2}$. . On signalera le modèle de Michaëlis-Menten et ses dérivés qui décrivent macroscopiquement les réactions biochimiques utilisées avec bonheur pour modéliser des processus cellulaires et des voies métaboliques. Cette cinétique est d'ailleurs démontrable à partir de réactions chimiques élémentaires faisant intervenir les " complexes enzyme-susbtrat ».

Or, non seulement les systèmes biologiques ne sont pas à l'équilibre (ce qui est le problème que l'on traite le plus facilement), mais ils sont toujours en interaction avec de multiples autres processus, dont très peu sont décrits à ce jour de façon complète, compliquant de ce fait les simulations déterministes. De plus, le vivant se caractérise par sa capacité à se reproduire, à se développer, et par son évolution, que l'on explique la plupart du temps a posteriori. Néanmoins, il existe des modèles dont l'utilisation est efficace dans de nombreux champs de la biologie. C'est le cas, par exemple, du modèle de Monod, dont le formalisme

\footnotetext{
'Rappelons, par exemple, que deux siècles séparent Isaac Newton de Louis Pasteur, et que la biologie moléculaire, qui peut laisser espérer la formalisation d'une " théorie du Vivant", comparable à celle des grandes théories physiques, appartient au dernier quart du $\mathrm{Xx}^{\mathrm{e}}$ siècle (Lecourt, 1999).

${ }^{2}$ La différence entre les réactions chimiques et biochimiques est que ces dernières sont catalysées par des protéines particulières que sont les enzymes.
} 
s'apparente à celui de Michaëlis-Menten. Il a été développé au départ pour décrire des croissances bactériennes en laboratoire, mais s'est avéré utile pour de nombreuses autres situations de dynamique des populations. On retiendra enfin que ce modèle est historiquement le premier en biologie qui introduit un véritable bilan entre la matière vivante produite (la biomasse) et les ressources consommées pour la produire.

La modélisation des processus biologiques reste donc, à ce jour, peu mécanistique et globalement empirique. La plupart des représentations conceptuelles et numériques reposent sur des bilans de matière (dans le meilleur des cas d'énergie) paramétrisés. Sauf exceptions ${ }^{3}$, ces paramètres ne sont pas directement accessibles à l'expérience mais doivent être identifiés au moyen de mesures plus intégrées. De manière générale, les étapes de calibration-validation sont donc incontournables et placent l'observation au cœur du travail du biogéochimiste, tout en introduisant un certain nombre de difficultés liées aux échelles d'expérimentation et de simulation.

Enfin, l'assemblage de ces différents processus, dans des milieux hors laboratoire, nécessaire à la compréhension des cycles biogéochimiques oblige à repenser les modèles précédents dans des contextes hétérogènes, non stationnaires et spatialisés. La modélisation doit alors prendre en compte des lois physiques, des niveaux d'organisation écologiques et les définitions d'échelles de temps et d'espace associés.

\section{Approche par éléments}

Le plan général du rapport montre bien les axes majeurs d'étude des cycles biogéochimiques. Ainsi l'approche par élément est encore la plus développée, avec de nombreux résultats que présentent les chapitres de la première partie. Il est intéressant de noter que, dans ces contributions, la modélisation intervient principalement par une approche conceptuelle, par exemple par des schémas en « boîtes et flèches " qui associent les divers compartiments physiques ${ }^{4}$ de la biosphère où interviennent les éléments en question.

Même si les dernières avancées (par exemple, colimitation de certaines formes de production primaire océanique par des éléments comme le fer, Mills et al., 2004), ne font que confirmer qu'il est illusoire de chercher à comprendre le cycle du carbone de façon autonome, il est cependant fondamental de remarquer que les échelles de temps et d'espace associées à ces descriptions sont implicitement liées à l'élément observé. Dans ce cas par exemple, le cycle du

\footnotetext{
${ }^{3}$ Par exemple, Servais, Billen et Hascoët, 1987.

${ }^{4}$ Par compartiment physique, on entend des boîtes spatiales 3D.
} 
carbone peut s'envisager à l'échelle géologique, alors que l'échelle temporelle des apports de fer atmosphérique par les poussières sahariennes est de quelques jours et que cette échelle varie encore avec le climat. . Des méthodologies adaptées doivent être mises en œuvre pour réaliser de telles intégrations.

Étudiée de longue date, l'eutrophisation des milieux lacustres a conduit à une approche couplée relativement précoce des cycles des nutriments (azote et phosphore) en intégrant la dimension biologique de la production primaire.

Sachant que les micro-organismes du compartiment sol en sont des acteurs essentiels (nitrifiants, fixateurs et dénitrifiants), la dynamique de cet élément a été suivie et modélisée simultanément à celle de ces différentes catégories de micro-organismes, aussi bien en laboratoire que sur le terrain. Les modèles ont pris explicitement en compte les processus impliqués. Cependant, les échelles de temps et d'espace restent limitées à celles du processus de laboratoire ou de la parcelle ${ }^{5}$.

\section{Approche par milieux}

Là encore, le plan est exemplaire, puisque si la première partie est plutôt dévolue aux éléments, la deuxième partie est principalement consacrée à une approche par milieux. Cette approche nécessite une intégration plus importante dans la modélisation puisqu'elle se concentre sur un système spatialisé lécosystème forestier, écosystème marin, écosystème estuarien, etc.). La modélisation de tels systèmes doit alors intégrer les principaux éléments chimiques intervenant aux échelles (temporelles et spatiales) du système étudié.

La complexité supplémentaire introduite par l'hétérogénéité des milieux permet d'expliquer la différence dans le développement de la modélisation des systèmes terrestres par rapport aux systèmes aquatiques. En effet, dans les systèmes aquatiques, la physique de l'écoulement joue généralement un rôle prédominant et assure le transport et le mélange des solutés. Pour l'océan par exemple, le milieu physique est soit plus homogène locéan profond, cf. les représentations de Wallace Broecker), soit l'on dispose d'outils plus ou moins complexes pour représenter ses hétérogénéités. Ainsi, la zone côtière, qui nécessite un maillage plus fin afin de correctement décrire les interactions entre la colonne d'eau et le fond à la méso-échelle, peut être représentée avec des modèles de type " couche limite " (Laugier et al., 1996; Taillandier et al., 2004). Les écosystèmes forestiers représentent une situation intermédiaire avant d'entrer dans une plus grande hétérogénéité pour le cas des écosystèmes terrestres anthropisés (terres agricoles,

\footnotetext{
${ }^{5} \mathrm{Cf}$. les travaux du laboratoire d'écologie microbienne de Lyon (Bardin, Normand) et de Wageningen (van Veen, 1977).
} 
systèmes périurbains). La mise en œuvre spatialisée du modèle agronomique STICS (voir encadré 9.1), par exemple, requiert un maillage à l'échelle de la parcelle agricole, dans la mesure où le type de culture conditionne la paramétrisation (Feller et al., 2004).

\section{Encadré 9.1}

\section{Un exemple de modélisation agronomique}

STICS (Simulateur mulTIdisciplinaire pour les Cultures Standard) est un modèle de fonctionnement des cultures conçu comme un outil de simulation opérationnel en conditions agricoles (Brisson et al., 1998). C'est le modèle retenu dans le thème 1 du Piren-Seine pour simuler le lessivage des nitrates. STICS simule les bilans d'eau, de carbone et d'azote dans le système solplante. II a été mis au point par diverses équipes de l'Inra afin de simuler la production agricole (en quantité et qualité) et les fuites de nitrate à l'échelle de la parcelle agricole et du cycle cultural, en fonction du climat, du type de sol, du type de culture et des pratiques agricoles. II résulte de l'assemblage de plusieurs sous-modèles testés antérieurement (Brisson et al., 1998). Il a d'abord été évalué en sol sans culture, et pour les cultures de blé d'hiver ef de maïs (Justes et al., 2001). Il a également été comparé favorablement à d'autres modèles proposés dans la littérature internationale (Gabrielle et al., 2002).

Le système sol simulé correspond à la zone d'enracinement maximale permise par le sol et la culture. Les flux d'eau et de nitrate sortant sous la base racinaire sont supposés alimenter de façon conservative les systèmes aquifères (zones non saturée et saturée). Les principaux processus simulés par ce modèle sont l'évaporation du sol, la transpiration de la plante, l'irrigation, le drainage naturel du sol; le développement, l'interception du rayonnement, la croissance foliaire, l'extension du système racinaire, la croissance en biomasse, l'élaboration du rendement de la culture; le transfert de nitrate dans le sol, l'absorption par le système racinaire, la fixation symbiotique, la décomposition des matières organiques, la minéralisation d'azote par le sol.

Ce modèle agronomique, initialement destiné à effectuer des simulations à l'échelle de la parcelle agricole, a déjà été employé de façon spatialisée, à l'aide du système d'information géographique Arc-Info, afin de simuler la pollution diffuse par les nitrates dans des bassins hydrologiques en zone de grande culture (Beaudoin et al., 1999; Schnebelen, 2000).

On considère que la culture est homogène et le système sol monodimensionnel. Son pas de temps est le jour. Il peut simuler un cycle cultural, mais peut aussi enchaîner les simulations sur un grand nombre d'années. Il faut alors 
définir la succession des cultures pendant cette période de temps. La mise en ceuvre du modèle requiert quatre sources de données :

- des données climatiques : Il s'agit de la température minimale, maximale, de la pluviométrie, de l'évapotranspiration potentielle (ETPI et du rayonnement global, au pas de temps journalier:

- des données \& sol " : ce sont les caractéristiques physicochimiques de la couche labourée lteneurs en argile, en calcaire, en azote organique, albédo,... I, les propriétés physiques de chacune des couches pédologiques lépaisseur, masse volumique, teneurs en eau à la capacité au champ et au point de flétrissement, teneur en cailloux) et la profondeur maximale d'enracinement permise par le sol,

- des paramètres « culture » : il s'agit des paramètres relatifs aux différents processus simulés Igermination, levée, développement, mise en place des capteurs souterrains et aériens, absorption d'azote, ..., incluant la prise en compte de l'effet des stress hydrique, thermique et azoté sur le fonctionnement de la plante;

- des données « techniques culturales » : elles sont définies par les modalités et les dates des interventions culturales (semis, irrigation, apports d'engrais azotés, apport d'amendements organiques, récolte, travail du sol, incorporation des résidus de culture, ....

Source: rapport de synthèse Piren-Seine 1998-2001, Gomes et al.

L'hétérogénéité des milieux cohésifs (sols, sédiments) est d'autant plus importante qu'ils sont le siège d'une activité biologique, voire humaine. Dans les sédiments, par exemple, on observe des micro-environnements oxiques, à l'échelle de quelques millimètres, qui sont la trace du passage de vers (Motelica-Heino et Davison, 2003; Davison et Motelica-Heino, 2002). Ces micro-environnements ont une importance capitale pour le cycle des métaux puisqu'ils permettent leur immobilisation sous forme d'oxydes. Cependant, ce n'est que très récemment que le développement de micro-électrodes déployables à l'interface eausédiment a rendu possible leur observation systématique (Viollier et al., 2003). La figure 9.1 présente une illustration des grandeurs caractéristiques de structuration du sédiment de la baie de Chesapeake selon l'importance respective du transport physique et de la bioturbation. On comprend que cette échelle d'observation ne pourra pas être reproduite de façon déterministe sur la seule base des lois physiques. 


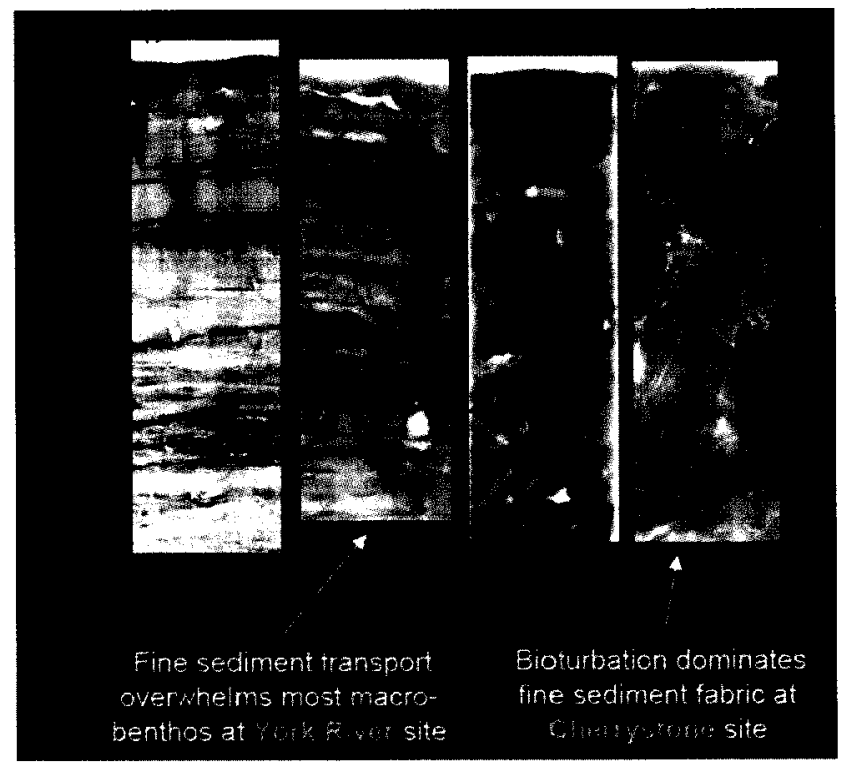

Vers l'aval de la baie.

Extrait de « Development of a Sediment Transport Model for the Chesapeake Bay : Supporting Physical data »; 2004, Lawrence P. Sanford, Carl T. Friedrichs, Jerome P.-Y. Maa.

Figure 9.1

Profils sédimentaires obtenus aux rayons $X$ sur différents sites de Chesapeake Bay. Au site amont, la dynomique sédimentaire est dominée par le transport de sédiments fins, formant des couches, alors qu'en aval, on distingue nettement les traces de passage des organismes benthiques dans un environnement par ailleurs homogène.

\section{Typologie sommaire des modèles}

\section{Modèles conceptuels - modèles formels ef fonctionnels}

Dans la littérature, on trouve plusieurs catégories de modèles. D'abord, ceux conceptuels exprimant les relations entre des entités. Ce sont pour la plupart des schémas purement relationnels. Les entités en question peuvent être des compartiments physiques (eau, sol, atmosphère), des espèces chimiques (divers composés de l'azotel, des organismes ou des populations biologiques. De nombreux schémas des chapitres 1 et 2 peuvent revendiquer ce statut. Ensuite les modèles formels et fonctionnels expriment la nature des relations par des objets mathématiques et de là peuvent être utilisés pour obtenir des résultats numériques, via des simulations (cf. figure 9.2, de $\mathrm{Cl}$. Schmidt-Lainé et A. Pavé, Environnement : modélisation et modèles pour comprendre, agir et décider dans un contexte interdisciplinaire. Natures-Sciences-Société : Sciences pour l'ingénierie de l'environnement 10 : s. 1, 2002, 5-25). 


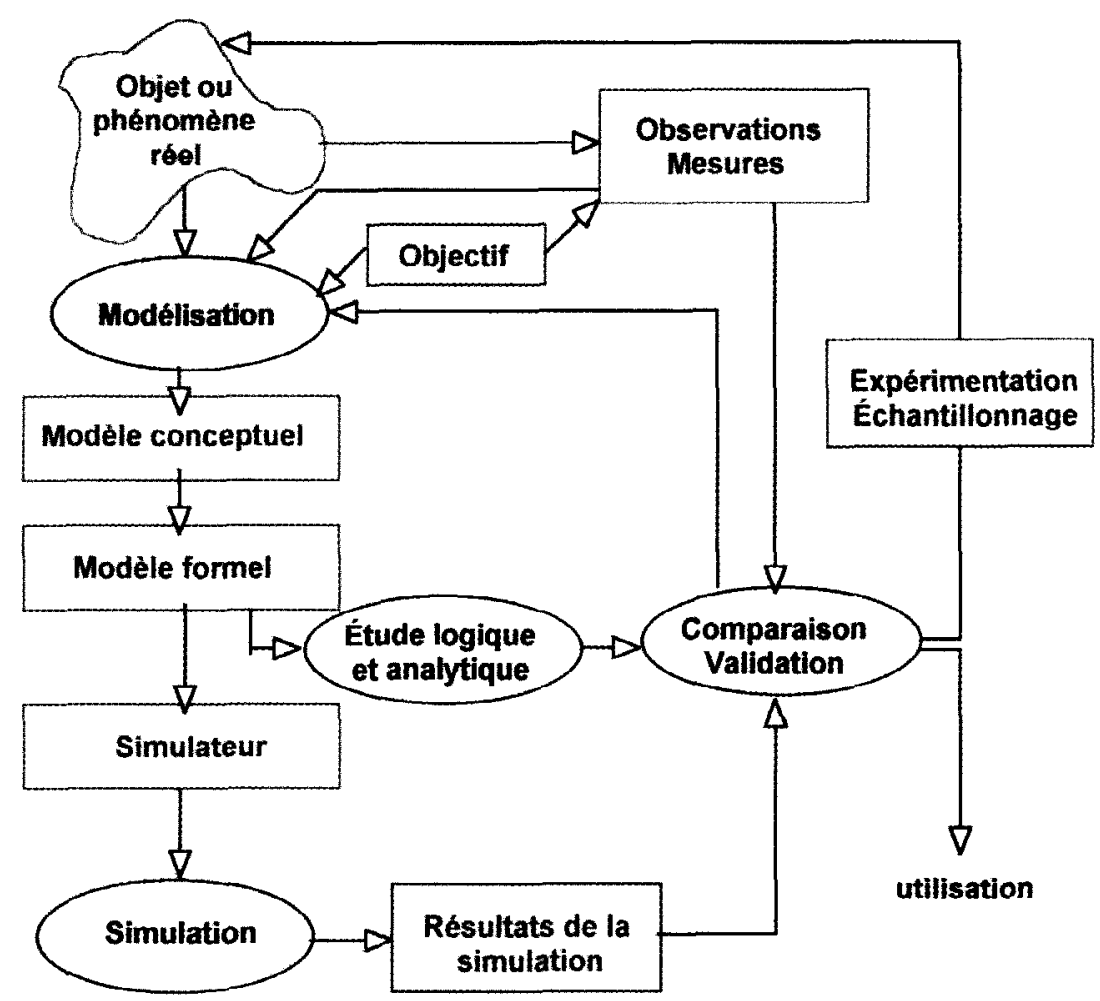

Figure 9.2

Schéma méthodologique général de modélisation ef de simulation. II y a lieu de bien distinguer les deux étapes : un modèle peut convenir ou ne pas convenir et le simulateur peut donner des résultats corrects ou incorrects. II y a donc bien lieu de procéder aux vérifications adéquates, notamment au bon fonctionnement du simulateur. II est aussi nécessaire de bien établir les relations avec les observations et l'expérimentation, quand elle est possible ("Models need data. Data need models»). Souvent, avant d'écrire un modèle formel, par exemple sous la forme d'une expression mathématique, on établit un modèle conceptuel sous une forme plus ou moins symbolique, par exemple sous la forme d'un diagramme en boîtes et flèches.

\section{Modeles formels et fonctionnels}

On distingue principalement deux catégories de modèles :

- les modèles non spatialisés qui vont représenter des relations quantitatives entre des « variables d'état » à l'intérieur de « compartiments » ainsi que les relations entre ces compartiments (par exemple, la quantité ou la concentration de carbone dans l'atmosphère, l'eau et le sol). Ces modèles permettent de simuler les variations de ces variables au cours du 
temps dans les divers compartiments. Les échelles à prendre en compte sont les échelles de temps et donc les grandeurs caractéristiques associées. (exemple : le modèle d'écosystème Rive, de Billen et al., 1994, qui est couplé à différents modèles de transport, Berner et al., 2003, 2004);

- les modèles spatialisés, qui vont décrire les variations de ces quantités aux divers points d'un espace, en l'occurrence un espace géographique. Dans ce cas, en plus des problèmes d'échelles de temps, se posent ceux qui sont relatifs au maillage de cet espace et donc d'échelle spatiale là l'échelle de la zone côtière ou de l'océan mondial par exemple, Tusseau-Vuillemin et al., 1998; Yool et Fasham, 2001);

- on trouve une catégorie intermédiaire où l'organisation spatiale est très sommaire, par exemple un découpage de l'océan en quelques boîtes (Broecker et Peng, 1982), ou bien un découpage latitudinal de la planète (Cao et Woodward, 1998).

Modèles et objectifs de la modélisation

On remarquera que les modèles peuvent être conçus à des fins différentes (connaissance, prévision, contrôle, estimation de certaines grandeurs non mesurables directement) et qu'il est nécessaire d'en tenir compte au moment de cette conception (choix des variables, des écritures des relations entre ces variables - les modèles élémentaires des processus -, des échelles de temps et d'espace, etc.). Enfin, toutes les démarches intégrant la modélisation se heurtent à des problèmes de cohérence entre les grandeurs caractéristiques des processus en cause, de pertinence spatiale et temporelle des variables d'état, donc à des problèmes de choix et de changement d'échelles de temps et d'espace. Ce point est examiné plus précisément dans ce qui suit.

\section{Grandeurs caractéristiques, échelles d'observation, maillage et approximation}

Un chapitre ${ }^{6}$ a été consacré aux questions d'échelles et de changement d'échelles dans le cadre de la modélisation dans le RST, n ${ }^{\circ} 15$ Études sur l'environnement : de l'échelle du territoire à celle du continent. Nous reprenons dans ce qui suit l'essentiel de ce chapitre méthodologique, dans le contexte de la modélisation des cycles biogéochimiques.

\footnotetext{
${ }^{6}$ André J-C., Mégie G., Schmidt-Lainé C. Échelles et changements d'échelles, problématiques et outils. In Caseau P. (ed). Études sur l'environnement : de l'échelle du territoire à celle du continent, RST, Académie des sciences, Tec \& Doc, Paris 2003, 167-199.
} 
La notion d'échelle est très largement répandue dans la littérature, avec des acceptions différentes suivant les disciplines. Dans les questions liées au changement global, c'est une notion très utilisée et finalement assez peu définie. Dans la thématique des cycles biogéochimiques, l'intégration de processus de natures très variées (physique, chimique, biologique, géologique, ...) à des niveaux de description très hétérogènes nécessite de préciser celte notion d'échelle.

On s'aperçoit en effet que ce terme est employé dans les cas suivants :

- échelle comme grandeur caractéristique d'un processus;

- échelle comme niveau d'observation et de représentation : échelle globale, échelle régionale, échelle locale;

- échelle comme maille d'approximation liée à l'instrumentation et à la simulation.

Nous précisons ces trois notions dans ce qui suit.

\subsection{Grandeurs caractéristiques}

Les échelles liées aux processus peuvent être définies comme des grandeurs minimales de description d'un phénomène. Pour cette raison, il est plus adapté d'employer le terme " grandeur caractéristique » qu'échelle. C'est l'échelon irréductible de description des phénomènes, le problème du passage du discret au continu, illustré par exemple en mécanique et en biologie par l'établissement :

- de grandeurs caractéristiques spatiales et temporelles par moyenne statistique en mécanique des fluides;

- de propriétés des tissus biologiques comme association de cellules.

On notera que les grandeurs caractéristiques liées à l'espace diffèrent suivant le type de processus. Pour les processus physiques au sens large, on déterminera des longueurs caractéristiques. Pour les processus biologiques ou économiques, on parlera plutôt de niveaux d'organisation, d'aires de répartition, de zones d'activité. Les grandeurs caractéristiques spatiales et temporelles ne sont pas indépendantes.

On renvoie au chapitre mentionné du RST N ${ }^{\circ} 15$ pour le détail des grandeurs caractéristiques spatiales et temporelles des différents processus intervenant dans les cycles biogéochimiques : processus hydrologiques, processus biologiques, sols et végétation, processus physicochimiques, processus et systèmes écologiques. 


\section{2 Échelles d'observation et de représentation}

Les échelles d'observation renvoient directement à la notion de représentation géographique ou de niveaux ou encore de compartiments d'étude. C'est pourquoi le mot échelle semble ici bien adapté pour désigner les trois niveaux désormais classiques : échelle globale, échelle régionale, échelle locale.

\subsection{1 Échelle globale}

C'est l'échelle de l'étude du climat et des grands systèmes (atmosphère, océans, continents). La maille de description est actuellement de l'ordre de $100 \mathrm{~km}^{2}$, mais il est envisageable de la porter prochainement à la dizaine de $\mathrm{km}$. Comme on le verra plus loin, ces valeurs ne sont pas dictées par une grandeur caractéristique d'un processus, mais sont complètement liées à l'approximation déterminée par les puissances de calcul à disposition.

Les nombreux processus entrant en jeu sont totalement dominés par la circulation générale des fluides et pris en compte d'un point de vue agrégé sous la forme de bilan d'énergie. Ce compartiment dominant fournit des forçages (température, vent, pression) aux niveaux régionaux et locaux. L'étude du changement global fait apparaître un temps caractéristique en multiples d'années - même s'il convient d'envisager des temps plus courts pour les études intraannuelles de saisonnalité, par exemple - alors que la désagrégation au niveau régional introduit de manière fondamentale la saisonnalité.

La description correspondante du climat à l'échelle globale nécessite la modélisation fine de processus atmosphériques, encore appelés "modèles de sousmailles ». Pour se restreindre aux seuls processus concernés ici, un des problèmes majeurs est l'obtention de descriptions satisfaisantes des échanges de chaleur et d'humidité entre d'une part l'océan ou les surfaces continentales, notamment le couvert végétal, et, d'autre part l'atmosphère. On touche ainsi la délicate question du changement d'échelle, traité au $\S 2$, et en particulier aux problèmes de hiérarchie. La climatologie est probablement le domaine scientifique où cette question a été prise en compte le plus tôt.

\subsection{2 Échelle régionale ou échelle des territoires}

La notion d'échelle étant liée à l'observation et à la représentation au sens géographique, il est naturel de rencontrer dans la littérature au moins deux types d'échelles régionales: 
- les échelles régionales climatiques $\left(c^{\prime}\right.$ est le sens du mot américain " regional ») que l'on peut appeler échelles continentales et qui correspondent à la désagrégation continentale de l'échelle globale;

- les échelles vraiment régionales (ou méso-échelles), telles que représentées par les systèmes décrits dans la section 2 du présent chapitre.

Ces échelles prennent en compte :

- les grandeurs caractéristiques liées aux différents processus en présence;

- l'organisation physique et topographique des écosystèmes à son meilleur niveau de cohérence (bassins versants, systèmes forestiers, systèmes littoraux);

- les niveaux d'organisation humaine dans ses activités (aménagement du territoire, agriculture, urbanisation).

L'échelle des écosystèmes contient donc bon nombre de grandeurs caractéristiques et prend en compte le point de vue humain avec ses propres grandeurs de référence, par exemple la génération humaine comme temps caractéristique.

Avec cette échelle, on est proche de la notion d'impact, dans le sens où les échelles de temps et d'espace sont liées à l'homme. C'est une notion directionnelle descendante qui s'intéresse de façon privilégiée au changement d'échelle global-régional par désagrégation et modélisation des processus.

La prise en compte des rétroactions est assez peu présente et met également en évidence les difficultés d'agrégation de certains processus décrits par ailleurs finement à l'échelle locale. En revanche, d'autres processus, comme la production de biomasse, se prêtent bien à de simples sommations sur une aire géographique (O'Neill, 1988).

C'est pourtant dans ce compartiment qu'interviennent le maximum de " couplages " et donc de problèmes liés au changement d'échelle. On retiendra de la théorie de la hiérarchie les deux critères qui sont probablement les plus représentatifs des difficultés de la modélisation à cette échelle (O'Neill, 1988) :

- la détermination de la bonne échelle régionale, au sens où les variables d'état relatives à une discipline dans un modèle sont aussi variables d'état pour une autre discipline dans un autre modèle;

- la cohérence de description des différents phénomènes en présence qui assure une modélisation à un niveau pertinent pour chaque processus. 
L'objectif étant de parvenir à une description complète, mais restant équilibrée et efficace, il est alors inutile d'interfacer des modèles dont les niveaux de description internes sont très différents. Les pistes pour décrire de plus en plus finement certains mécanismes ne vont en particulier pas dans le sens de l'amélioration des connaissances au niveau intégré.

Il faut également signaler dans l'interface des processus à l'échelle régionale la difficile harmonisation des couples de longueurs et de temps caractéristiques. Dans la plupart des cas, on remarque que les échelles spatiales sont privilégiées au détriment des temps de référence.

\subsection{3 Échelle locale}

C'est l'échelle du processus, qu'il soit biologique ou physicochimique. Dans ce cas, l'échelle d'observation est du même ordre que les grandeurs caractéristiques du mécanisme étudié. À cette échelle, certains des processus apparaissent comme discrets, ce qui n'est jamais le cas aux échelles supérieures. C'est donc au niveau local que l'on doit résoudre le problème de l'homogénéisation, $c^{\prime}$ està-dire le passage du discret au continu.

La question importante est ici la remontée d'informations à l'échelle régionale. On renvoie à l'exemple de l'évaluation des échanges ponctuels forêt-atmosphère (Saugier, 1997; Schmidt-Lainé, 1999).

En effet, même s'il semble plus naturel de décrire chaque processus et d'agréger les résultats pour l'étude de l'échelon régional, celte transmission hiérarchique est pourtant plus difficile. On rappelle que ces emboîtements sont maîtrisés dans les sciences physiques liées au climat. Pour les processus biologiques, cette étape d'agrégation est encore souvent délicate, sauf à caractériser brutalement le phénomène par son équivalent physique à l'échelon supérieur (exemple de l'indice foliaire ne tenant pas compte de l'écophysiologie).

\subsection{Maillage et instrumentation}

La collecte des données, l'obtention de résultats numériques et leur représentation spatialisée induisent à leur tour des grandeurs limitantes parfois confondues avec la notion d'échelle d'observation ou de grandeur caractéristique (dans ce contexte, on pourrait parler de plus petit niveau de spatialisation). Or, rappelons que la confrontation des résultats de modélisation biogéochimique avec les données est au cœur de l'étape de validation des paramètres 
empiriquement évalués, et préalable à toute utilisation du modèle en conditions prédictives.

On pense par exemple à la maille minimale actuelle de calcul dans le problème du climat global ( $100 \mathrm{~km}$ ), contrainte par la puissance calcul et non pas justifiée par la physique du phénomène. On peut également citer comme facteur limitant la résolution des images satellitaires décrivant l'hydrologie, la couverture végétale du territoire ou bien les champs de température et de chlorophylle océaniques. De même, l'échantillonnage dans la répartition des différents capteurs induit une maille minimale de description fiable des phénomènes. C'est un vrai problème encore susceptible d'amélioration, si l'on considère par exemple la répartition des points de mesure mondiaux d'échanges forêt-atmosphère dans le réseau Fluxnet.

À l'opposé, les récents développements technologiques qui ont conduit au déploiement de nouveaux microcapteurs in situ, sans perturbation du milieu, permettent d'acquérir des données électrochimiques à très petite échelle (submillimétrique) et pratiquement en continu (Viollier et al., 2003). L'utilisation de ces données en modélisation posera très probablement des questions méthodologiques.

Tous ces problèmes ont en commun le fait qu'ils demandent un compromis entre la qualité de la représentation et les ressources disponibles. La notion de maille semble alors être la bonne terminologie pour désigner ces différents problèmes d'approximation.

\section{2 | Changements d'échelles, couplages et données}

\subsection{Hiérarchie}

La problématique du "changement d'échelles " telle qu'employée dans la littérature, par exemple la transmission d'information d'une échelle d'observation à une autre, est fortement liée à la notion de hiérarchie. Par exemple, les systèmes biologiques sont organisés en niveaux dans une organisation hiérarchique. Ainsi un organisme, un individu, une population fournissent des exemples de niveaux. La théorie de la hiérarchie (O'Neill, 1988) généralise le concept en suggérant que tout processus à une certaine échelle d'observation est contraint par les conditions environnementales des niveaux voisins (au-dessus et en dessous). Les questions principales se concentrent sur la détermination d'une " hiérarchie » fondamentale et du niveau de référence pour un phénomène (on pourrait dire ici qu'il s'agit de déterminer ses grandeurs caractéristiques). 
On rappelle les grandes lignes des principaux résultats de cette théorie :

- les principes ou modèles d'interactions ne se transportent pas d'un niveau à un autre ;

- le niveau supérieur influe sur le niveau inférieur en transmettant des données quasi stationnaires; en effet, les processus du niveau supérieur ont des grandeurs caractéristiques plus grandes en temps et en espace, et sont donc plus lents;

- la remontée d'informations du niveau inférieur au niveau supérieur est plus délicate, bien que semblant plus naturelle ${ }^{8}$.

Dans tous les cas, le point important reste la détermination du bon niveau d'observation permettant de décrire de façon cohérente les différents processus.

Les questions relatives aux hiérarchies et aux emboîtements d'échelles d'observation ont fait l'objet d'études détaillées dans le domaine de la climatologie. On renvoie au $\$ 2.2$ «agrégation 》 (remontée d'information) et au $\$ 2.3$ « désagrégation » (descente d'information). La question similaire dans les sciences du vivant $n^{\prime}$ a été envisagée sérieusement qu'à partir du moment où l'écologie s'est développée. En effet, l'étude de la transmission d'un niveau à un autre est la question centrale de cette discipline.

Rappelons enfin que les processus intégrés décrits à l'échelle globale privilégient les flux et les densités, c'est-à-dire les grandeurs physiques, alors qu'ils sont beaucoup plus « orientés individus » dans une description à l'échelle locale.

\subsection{Agrégation, modèles bottom up}

C'est la démarche naturelle de remontée à une échelle supérieure d'informations sur des processus dont la description s'opère à plus petites grandeurs caractéristiques.

\footnotetext{
${ }^{7}$ Sauf dans le cas d'autosimilarité.

${ }^{8}$ La difficulté provient de ce qu'a indiqué le premier alinéa. Les relations entre des valeurs moyennes ne sont (en général) pas les moyennes des relations: exemple de la turbulence.
} 


\subsubsection{Agrégation et méthodes statistiques}

L'agrégation el l'homogénéisation font partie des techniques de base de la modélisation. Elles sont utilisées de manière systématique en physique et en science des matériaux. Pour passer à une échelle supérieure, on " paramétrise ", c'est-à-dire que l'on représente de façon statistique ce qui est trop petit pour être décrit explicitement à la maille du modèle de simulation avec lequel on veut travailler, à partir d'approches différentes et/ou complémentaires :

- une connaissance a priori des microphénomènes : pour reprendre l'exemple de la régulation stomatique, on sait que les stomates se ferment en cas de stress hydrique, on "paramétrise " donc à l'échelle supérieure en utilisant un coefficient régulateur de l'évapotranspiration en fonction de la disponibilité en eau du sol (facteur bêta);

- une moyenne réalisée à partir de phénomènes individuels, soit observés (on fait des campagnes expérimentales pour observer finement), soit simulés à l'occasion d'expériences numériques fines à très haute résolution. Dans un cas comme dans l'autre, on moyenne ensuite les résultats.

C'est dans une telle approche que l'on voit ressurgir toute la difficulté liée aux non-linéarités (si l'on n'avait à moyenner que des phénomènes de base linéaires, la vie serait trop facile!). II faut tenir compte en particulier des effets de seuil qui se produisent à l'échelle locale et qui se traduisent par des effets analogues ou non à l'échelle du territoire. De façon générale, on est conduit à introduire des « relations de fermeture " du modèle, relations qui ne peuvent pas se déduire de la modélisation au niveau inférieur, et qui doivent être fondées sur l'expérimentation. Notons que l'approche "paramétrisation " est une approche où l'on dispose maintenant d'une stratégie de validation assez bien établie.

On peut aussi parler des inventaires, où l'idée est de reconstruire des quantités agrégées à partir d'estimations ponctuelles (les inventaires des sources d'émission des gaz à effet de serre et des puits de captage de ces gaz). Dans ce cas précis, on peut s'appuyer sur une méthode d'estimation complémentaire en se servant $d^{\prime}$ 'autres variables elles-mêmes intégratrices par nature : la consommation d'énergie fossile, qui détermine le total des émissions de $\mathrm{CO}_{2}$, le débit à l'exutoire d'un bassin versant, qui intègre toute (ou presque) l'hydrologie sur son territoire. II faudrait souligner tout l'intérêt à travailler avec des variables intégratrices de contrôle, même si ce n'est pas toujours automatiquement possible. 


\subsubsection{Modèles bottom-up}

Il s'agit en général de modèles où l'on renonce à créer des agrégats statistiques, et qui utilisent, de bout en bout, la même description fine des processus. C'est évidemment la description la plus satisfaisante pour coller au plus près au phénomène, mais il faut pouvoir remonter ces modèles à des échelles plus grandes et surtout intégrer les différents processus ainsi modélisés dans un système. De nombreuses discussions critiques ont lieu dans la littérature - notamment à propos des processus physiologiques - sur la validité de l'emploi de tels modèles, pourtant satisfaisants pour l'esprit. La première critique tient à la construction de ces modèles dont la validation, la calibration, la transparence ne sont pas encore assurées et ne pourront peut-être jamais l'être. La deuxième critique tient à l'imprécision des résultats issus de ces modèles d'un point de vue plus conceptuel de l'emboîtement des échelles. Selon de Wit (1978), il n'est pas raisonnable d'aller au-delà de modèles à deux étages reliant trois échelles adjacentes. Citons le modèle biogéochimique Prose (Even et al., 1998), développé dans le Piren-Seine, qui résulte du couplage d'un modèle très détaillé du fonctionnement de l'écosystème aquatique (Rive) avec un modèle de transport dynamique unidimensionnel. Ce modèle est utilisé pour établir des bilans de matière à l'échelle du bief (par exemple, le Grand Morin : Flipo et al., 2004), alors que Rive comporte, selon les versions, de vingt-cinq à trente variables, et décrit jusqu'à la synthèse de métabolites par le phytoplancton (Lancelot et al., 1985), avec toutes les difficultés numériques que cela implique! Le même module biogéochimique, couplé à un autre modèle de transport hydrologique, permet de faire des simulations du développement phytoplanctonique à l'échelle du bassin de la Seine dans son ensemble (Billen et al., 1994).

\section{Encadré 9.2}

\section{Un exemple : simuler les variations spatiotemporelles de la réponse diagénétique ${ }^{9}$ des sédiments côtiers aux flux de matière issus de la colonne d'eau (Soetaert et al, 2000).}

La prise en comple de la réponse diagénétique aux flux de matière pélagique est indispensable à la compréhension du fonctionnement des zones côtières (Tusseau-Vuillemin et al., 1998). En effet, la profondeur y est peu importante; cela permet d'une part que la matière organique atteigne le fond avant d'avoir été reminéralisée par les micro-organismes pélagiques, cela permet également que les flux de nutriments issus de la diagenèse sédimentaire aient un impact significatif en retour sur la production primaire

\footnotetext{
${ }^{9}$ La diagenèse est l'ensemble des changements biochimiques et physicochimiques affectant un sédiment après son dépôt, avant qu'il ne passe en domaine métamorphique.
} 
pélagique. Or, les échelles temporelles de ces différents processus Idiagenèse sédimentaire et production primaire pélagique) sont totalement différentes: de l'ordre de l'année pour la première, de la journée pour la deuxième. De fait, les modèles biogéochimiques adaptés à chacune des problématique sont généralement développés de façon séparée (Soetaert et al., 1996; Tusseau et al., 1997).

Soetaert et al. (2000) ont permis de coupler de facon dynamique les deux approches en paramétrisant la réponse diagénétique aux flux pélagiques. Au moyen d'une simulation stafistique de type Monte-Carlo, la réponse diagénétique a été simulée avec un modèle complexe (figure 9.3) et validé. Une base de données représentative des conditions océaniques a ainsi été créée, à partir de laquelle la réponse diagénétique peut être extrapolée instantanément en réponse à des flux pélagiques. La totalité de l'information contenue dans le modèle diagenétique complet est ainsi utilisée, sans que les temps de simulation soient prohibitits. Ce «métamodèle " a pu être utilisé pour simuler l'ensemble du Golfe du Lion (Méditerranée) en trois dimensions (Bonnet et al, 2000 ).

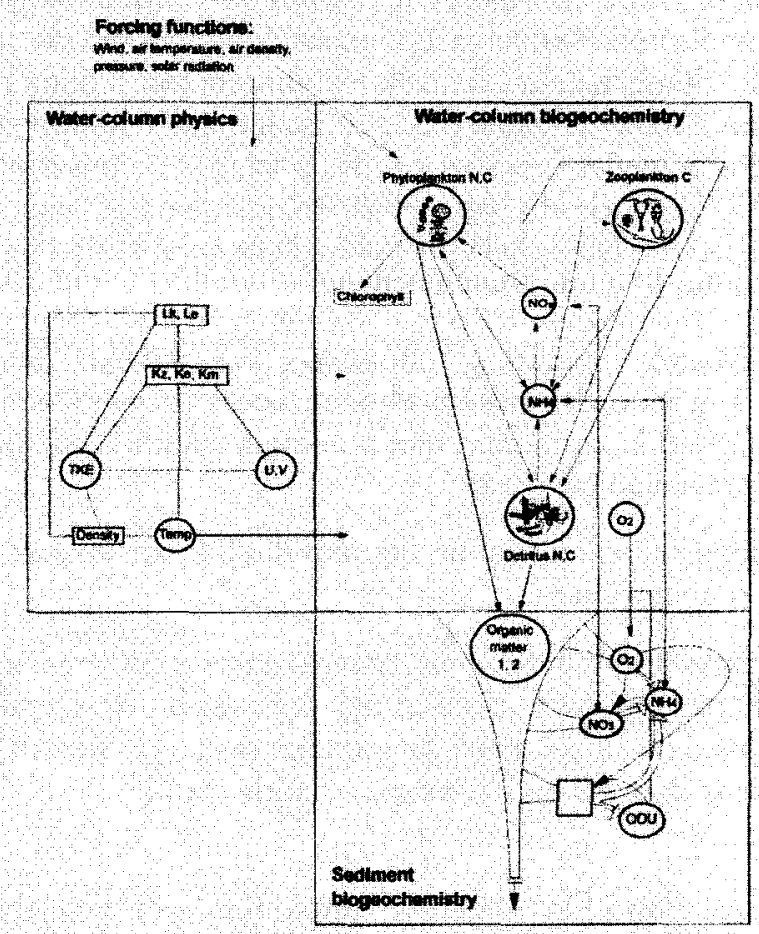

Figure 9.3

Représentation schématique du couplage entre les processus physiques et biogéochimiques de la colonne d'eau et la diagenèse du sédiment (Soetcert et al, 2000). 


\subsection{Désagrégation, modèles top down}

Ces méthodes sont particulièrement développées dans les modèles " physiques " liés au climat. De par leur antériorité et la nature physique dominante des modèles, des techniques plus complexes ont été mises au point qui permettent de passer du compartiment global au compartiment régional. Ces techniques se transmettent progressivement aux autres domaines de la biogéochimie, tout particulièrement pour les modèles spatialisés.

- La méthode de l'inversion climatique consistant à déduire la valeur locale $d^{\prime}$ une variable de sa valeur moyenne et de données locales. C'est la plus simple des méthodes de désagrégation spatiale. La modélisation inverse est souvent utilisée pour reconstituer les variations spatiales continues d'un ensemble de données discrètes, comme le sont naturellement les données collectées par exemple en océanographie (hors données satellitaires). Les champs obtenus ainsi sont utilisés pour initialiser les modèles ou bien pour leur validation. Citons Denis-Karafistan et al., (1998) et Karafistan et al., (2002) pour la reconstitution des champs de concentration de nitrates ef de phosphates en Méditerranée, grâce à un modèle de circulation générale. La désagrégation de l'information contenue dans les images de couleur de l'océan (images CZCS) a donné lieu à des modélisations inverses plus poussées reposant sur des principes de bio-optique (Morel et André, 1991); Bricaud et al., 1999). II s'agit de reconstituer des profils de pigments (ici la chlorophylle) à partir d'une information intégrée perçue par le satellite (Campbell et al., 2002).

- Les méthodes analogiques qui nécessitent un ensemble d'observations historiques à grande et petite échelles. L'objectif est de déterminer le climat local associé à la circulation atmosphérique globale simulée par un modèle de circulation générale.

- Les méthodes linéaires, qui ne peuvent décrire que la part de variabilité liée à la grande échelle.

- Les méthodes déterministes non linéaires comme les réseaux de neurones. Ces méthodes sont bien adaptées aux données bruitées, mais le caractère déterministe fournit des séries temporelles qui sous-estiment en particulier les événements extrêmes (fortes pluies, sécheresse).

Les modèles top down sont en général empiriques et contiennent donc peu de description de mécanismes. Les sorties du modèle sont contraintes par le principe adopté, qui veut que le comportement de la partie reproduise celui du tout, de sorte que, contrairement aux modèles bottom up, ils ne peuvent pas aboutir à de nouveaux types de comportements. On peut citer par exemple le modèle 
de Monteith (la croissance de la parcelle est une fonction du rayonnement absorbé), et le modèle de Priestley-Taylor (l'évapotranspiration est une fonction du rayonnement absorbé à l'échelle régionale).

\subsection{Couplage et interfaçage}

Le couplage désigne à la fois l'interfaçage de domaines physiques contigus décrits par des processus différents - typiquement le couplage océanatmosphère (Lions et al, 1992) - et le couplage de processus différents sur un même domaine (couplage carbone-climat dans les études sur les changements globauxl.

Les couplages spatiaux comportent de sérieuses difficultés techniques, mais ne présentent pas de problèmes conceptuels fondamentaux (figure 9.4), même si leur résolution peut s'avérer fastidieuse. Par exemple, pour les problèmes de raccordement, une importante méthodologie mathématique permet de modéliser au mieux les transitions (frontières artificielles, conditions aux limites transparentes ou absorbantes, assimilation). On a déjà cité plus haut les modèles de couche limite qui permettent d'améliorer le maillage dans une zone limitée du domaine et qui se développent particulièrement en océanographie (Laugier et al., 2004). Reprenons l'exemple du couplage de STICS avec MODCOU dans le cadre du Piren-Seine : le maillage final 7900 mailles pour le bassin de la Seine) résulte du croisement entre le maillage des deux modèles, lui-même contraint par les cartes des petites régions agricoles, des zones pédologiques et la grille de Météo-France.

En revanche, le couplage dans un même domaine de processus de natures différentes pose des vrais problèmes conceptuels. Il est en effet des domaines où la modélisation couplée ne s'obtient pas simplement par un interfaçage organisant la coexistence de sous-modèles. On rejoint ici la difficulté de la construction de modèles totalement couplés, intégrés, idéaux, qualifiés de " chimères " (Lions, 1997). Ce genre de couplage nécessite une analyse macroscopique permettant de dégager une hiérarchie de modèles. Cette hiérarchisation doit inclure une identification des échanges entre les différents modèles et sous-systèmes, qu'il s'agisse, dans le cas conceptuellement le plus simple, d'échanges par flux de matière et/ou d'énergie, ou, pour les cas plus complexes, d'échanges d'informations de nature plus morphologique, voire simplement de nature qualitative. C'est la démarche adoptée dans l'exemple cité plus haut de couplage entre la réponse diagénétique des sédiments et le flux de matière issu de la colonne d'eau. Ce n'est que lorsque les modes d'interaction, entre sous-modèles et soussystèmes, sont ainsi identifiés que la modélisation plus globale par couplage peut réellement se mettre en place. 


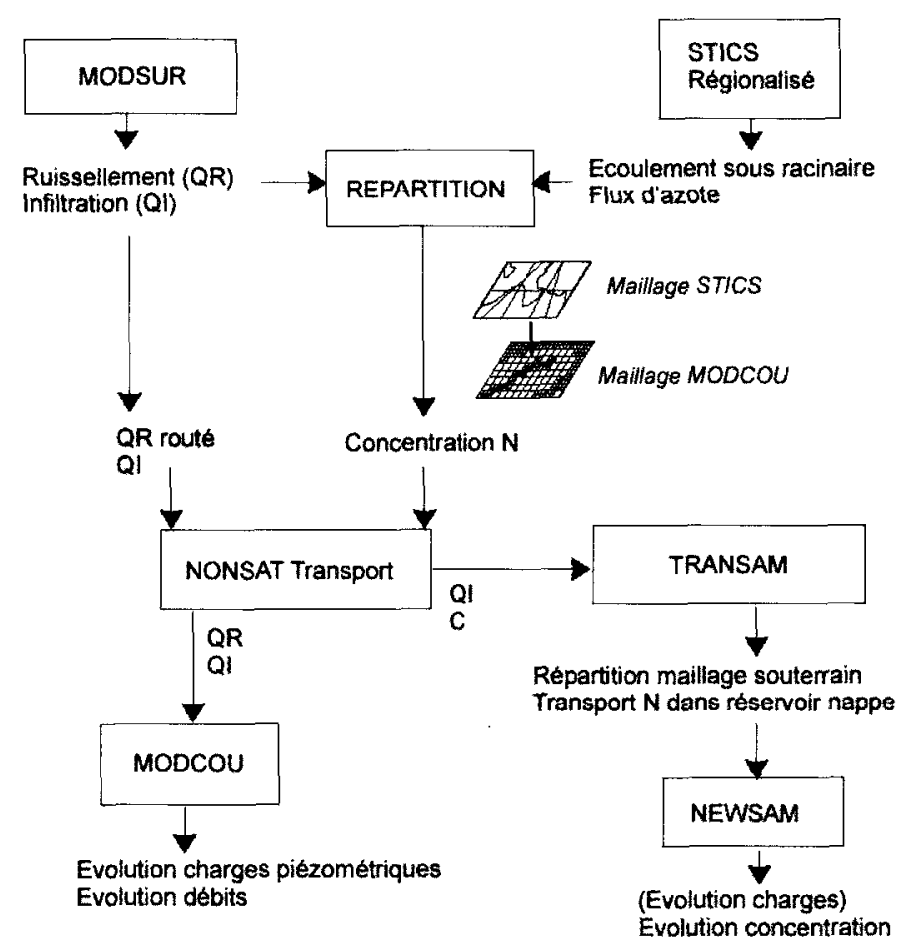

Figure 9.4

Représentation schématique du couplage entre les modèles hydrologiques ef biogéochimiques du Piren-Seine pour la simulation des flux d'azote diffus.

\subsection{Données}

On ne donne ici que les quelques éléments plus spécifiquement relatifs aux échelles.

\subsubsection{Validité et incertitude des données}

Les données d'observation sont toujours entachées d'erreurs et d'incertitude. $C^{\prime}$ est lorsque l'on s'intéresse aux échelles plus fines que la détection de ces erreurs, et donc leur contrôle, se révèle les plus délicats. Si en effet la météorologie globale sait utiliser au mieux les différentes régularités du système pour contrôler ses données grâce aux méthodes d'assimilation qui permettent d'exploiter les synergies et redondances physiques des données thermiques et dynamiques, à travers leur interaction dans un modèle (voir encadré), ces contrôles mutuels sont de plus en plus délicats à mettre en évidence et à exploiter, lorsque l'on s'intéresse à l'échelle régionale. En ce sens, le problème de la qualité des données se 
pose avec plus d'acuité à ces échelles. De plus, lorsque les données destinées à I'initialisation ou à la validation des modèles sont elles-mêmes issues d'un processus de désagrégation (cf. l'exemple cité plus haut des champs de chlorophylle issus des images CZCS), les hypothèses de la modélisation viennent s'ajouter à ces facteurs d'incertitude.

\section{Assimilation de données}

L'assimilation de données vise à optimiser l'information provenant du modèle et des observations, en sachant que chaque information est entachée d'erreur. L'assimilation de données permet de prendre en compte "plus de physique " dans l'algorithmique d'analyse de données et ainsi viser des performances métrologiques accrues pour les systèmes de traitement. Il n'y a donc pas de frontière absolve entre traitement et assimilation de données. Les deux grandes familles de méthodes d'assimilation de données (à savoir méthodes variationnelles ef méthodes séquentielles) connaissent toujours des développements importants. On peut en particulier citer les méthodes variationnelles du second ordre (qui permettent de calculer des sensibilités du modèle en présence de donnéesl, les avancées récentes concernant les extensions du filtrage de Kalman (applications aux modèles non linéaires, réduction des coûts de calcul liés à la matrice de covariance) et celles des méthodes de filtrage particulaire. Dans un cadre applicatif spécifique, il est nécessaire de mener une réflexion approfondie pour identifier les méthodes les plus adaptées aux modèles concernés, et plus généralement au " cahier des charges " qui motive la démarche. Dans certains cas, on peut ainsi par exemple vouloir optimiser la configuration du réseau d'observation (y compris éventuellement en temps réel lorsque l'on dispose de capteurs mobiles), ou s'intéresser à la propagation des incertitudes dans l'estimation. On peut se reporter à J. Blum (Hoteit et al., 2003) et F.X. Le Dimet (Le Dimet et al., 2002) pour les développements méthodologiques et à P. Prunet et JF. Minster (1996a, 1996b) pour une application à la biogéochimie.

\subsubsection{Dispositif de collectes de données el cohérence}

Au-delà de ce problème de qualité des données se pose de façon tout aussi cruciale celvi de l'échantillonnage et de la résolution spatiale et temporelle nécessaires pour décrire les différents phénomènes se déroulant à l'échelle des écosystèmes. Les dispositifs de collectes de données ne sont en effet pas toujours cohérents avec les échelles caractéristiques des quantités recueillies. Ceci est vrai dans de nombreux domaines, même si l'on ne cite ici que deux exemples:

- celui des forêts, où le travail traditionnel par « placettes » ne permet pas de bien appréhender la dynamique plus générale; 
- celui de l'estimation des flux de carbone émis au-dessus d'un territoire donné à l'échelle régionale, pour lequel il n'existe pas de mesures en réseaux à partir desquelles il serait possible d'agréger une valeur à l'échelle du système.

Il faut enfin signaler le problème du partage et de la mise à disposition de ces données de façon complète, cohérente et organisée à l'échelle des écosystèmes. Un effort en ce sens doit être mentionné dans le cadre de l'Observatoire de recherche en environnement F-ORE-T (chapitre 10.2). Une des opérations phare de cet ORE est la mise en place d'un système d'information destiné à faciliter celte question de partage des données.

\section{Évaluation des modèles biogéochimiques.}

George B. Arhonditsis et Michael Brett ont publié, en 2004, une très intéressante (et unique en son genre) synthèse sur la modélisation biogéochimique et mécanistique des milieux aquatiques. À partir de 153 études, variables par leur complexité, leurs échelles d'analyse et de simulation, voire leurs objectifs scientifiques et techniques, les auteurs tentent une évaluation cohérente de l'état de l'art de la discipline. Une grande majorité de ces publications ne fournissent pas de critère quantitatif de la qualité de la simulation (figure 9.5). Les auteurs l'ont évaluée eux-mêmes en digitalisant les graphes comparant simulation et données, ce qui demande toute une réflexion sur la résolution spatiale et temporelle à laquelle on effectue cette comparaison.

Peu d'auteurs utilisent des techniques d'optimisation, ce qui tient très probablement, non seulement à la culture «naturaliste » de la discipline, mais aussi au fait que certains des nombreux paramètres nécessitant calibration ne sont pas identifiables. Pour ces paramètres, qui sont généralement nécessaires à la cohérence de la modélisation, l'estimation par ajustement visuel ou à dires d'experts reste la plus couramment pratiquée. La validation reste également peu pratiquée (figure 9.5), alors que la paramétrisation la rend absolument nécessaire. L'une des raisons principales à cela est sans doute le manque de données, tout particulièrement pour les modèles spatialisés. Cependant, les auteurs de cette synthèse notent également que beaucoup d'études en modélisation biogéochimique pèchent par une définition trop imprécise de leurs objectifs a priori, ce qui conditionne les étapes de validation. Il faut également relever que les performances des modèles biogéochimiques sont rarement excellentes (du moins au vu des critères classiques d'évaluation, figure 9.6), ce qui encourage peut-être certains scientifiques à rester dans le flou quant à la validation. La synthèse de George Arhonditsis et Michael Brett permet de quantifier avec des indicateurs 
(a) Sensitivity analysis (\%)

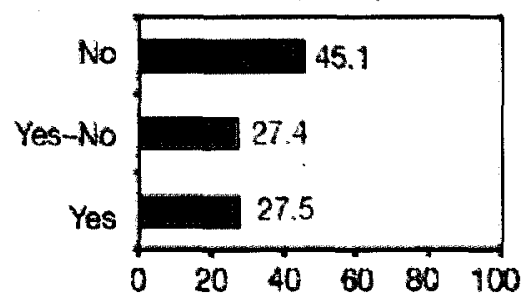

(c) Quantification of goodness-of-fit (\%)

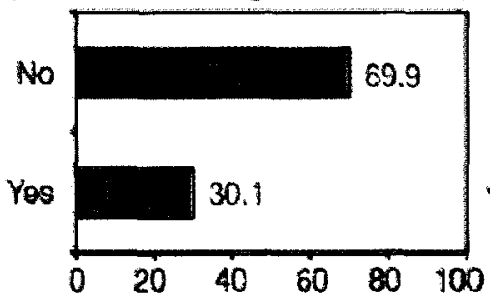

(b) Optimization (\%)

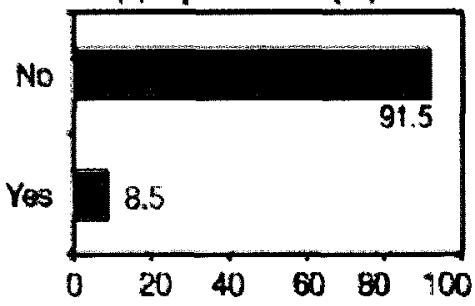

(d) Validation (\%)

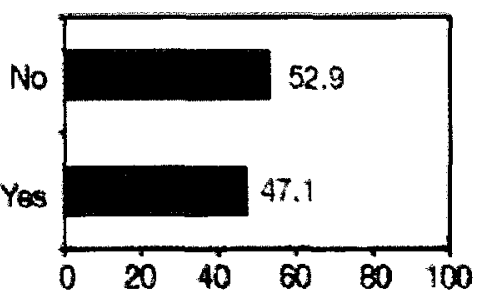

\section{Figure 9.5}

Proportions d'éfudes de modélisation biogéochimique aquatique (a) comportant une analyse de sensibilité, (b) ufilisant une technique d'optimisation pour la calibration du modèle, (c) quantifiant la qualité de l'adéquation entre simulation ef données, (d) comportant une validation du modèle.

factuels le décalage déjà évoqué entre la modélisation physique et biogéochimique. Il est en effet tout à fait frappant de constater que la qualité de reproduction des données se dégrade selon un axe inorganique-organique complexe (figure 9.6). Alors que la température et la concentration en oxygène dissous sont généralement bien reproduites, la dynamique du zooplancton et des bactéries reste difficile à simuler. Ces compartiments biologiques représentent un niveau de complexité supérieur au phytoplancton et dépendent $d^{\prime}$ autres niveaux biologiques. Enfin, soulignons avec les auteurs que les observations biogéochimiques ne devraient pas constituer l'unique critère d'évaluation des modèles (Evans et al., 2003), dans la mesure où elles ne représentent qu'une approximation de la complexité du vivant. Cela est particulièrement vrai pour le compartiment bactérien par exemple, dont l'observation est actuellement révolutionnée avec les techniques moléculaires. L'une des perspectives pour l'amélioration de ces modèles biogéochimiques serait la généralisation de l'assimilation de données, qui pourrait permettre de réduire les divergences progressives des simulations.

\section{Conclusion et recommandations}

\section{Partager une culture commune de la modélisation}

Comme rappelé dans l'introduction de ce chapitre, les différentes disciplines convoquées dans l'étude des cycles biogéochimiques n'ont pas la même 

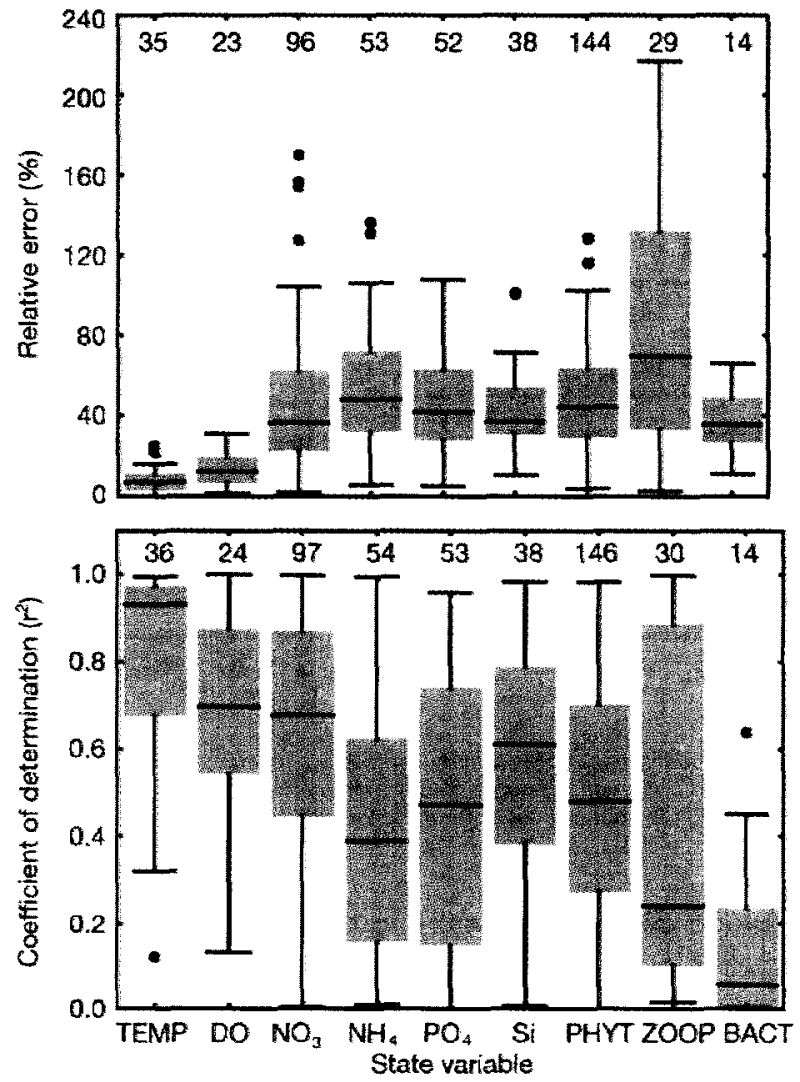

\section{Figure 9.6}

Performance des modèles aquatiques biogéochimiques étudiés. Erreurs relatives (\%) ef coefficients de détermination pour la température (TEMP), l'oxygène dissous (DO), les nitrates $\left(\mathrm{NO}_{3}\right)$, l'ammonium $\left(\mathrm{NH}_{4}\right)$, les phosphates $\left(\mathrm{PO}_{4}\right)$, la silice (Si), le phytoplancton (PHYT), le zooplancton (ZOO) et les bactéries (BACT). Le nombre d'études évaluées est indiqué pour chaque variable.

pratique de la modélisation. En particulier l'approche des processus biologiques, très souvent empirique et de surcroît non linéaire, n'a probablement pas encore trouvé sa juste place comme champ d'application des mathématiques appliquées. En effet, certaines techniques (optimisations complexes, résolution de systèmes complexes, etc.) sont couramment employées dans d'autres disciplines, plus portées sur des modèles mécanistes et plus proches des mathématiques. Cette appropriation, qui peut se faire notamment par le développement de thèses pluridisciplinaires en co-encadrement, pourrait dans certains cas accélérer la formalisation de processus, en évitant des redondances dans le développement des techniques, et faciliter le couplage avec les processus physiques. 


\section{Vers des modèles spatialisés}

L'étude par élément, qui a largement été développée dans l'étude des cycles biogéochimiques, prend le plus souvent en compte l'inhomogénéité spatiale des milieux par une approche de type « calage ». II est clair que l'approche par milieux nécessite une meilleure description spatiale des phénomènes, notamment pour expliciter les interactions chimie-biologie. Le développement de modèles spatialisés est donc une priorité qui renvoie à la possibilité de validation de ces modèles et d'obtention des données.

\section{La nécessaire collaboration données-observations-modélisation}

Les exemples développés ci-dessus, ainsi que l'encadré 9.3, portant sur l'étude de la dynamique des masses océaniques basée sur l'utilisation de marqueurs radioactifs, montrent clairement qu'il ne s'agit plus d'opposer expérimentation et modélisation mais bien de faire collaborer l'ensemble du dispositif données-observations-modélisation. L'exemple de l'assimilation de données est peut-être le plus illustratif : les données ainsi que les modèles sont entachés d'erreur. La collaboration données-modèles peut permettre de "recaler 》 les données pour autant que les modèles sont suffisamment avancés dans la prise en compte des mécanismes "physiques" au sens large. II faut souligner que les grandeurs biogéochimiques sont pour la plupart moins précisément mesurables que les grandeurs physiques. Outre la variabilité biologique, qui confère aux systèmes une importante incertitude structurelle, les analyses chimiques fournissent rarement des résultats absolus. Les chaînes analytiques deviennent parallèlement de plus en plus complexes, la mise au point des protocoles constituant des enjeux de recherche lexemple : chaînes chromatographiques doublées de deux spectromètres de masse pour mesurer des molécules organiques à l'état de trace dans le milieu naturel). La structure même de ces données biogéochimiques, avec leur degré d'incertitude et de variabilité, mériterait également d'être une source de développements méthodologiques nouveaux en modélisation pour la prise en compte de cette spécificité.

\section{Expliciter les échelles pour capitaliser les résultats de modèles}

Suivant le but de l'étude (analyse de processus ou bilan à grande échelle par exemple), l'échelle spatiale et donc temporelle peut être très différente. L'étude des publications relatives à ces modèles montre que ces échelles ne sont que très rarement explicitées, ce qui rend difficile, voire impossible, une capitalisation des résultats pour le changement d'échelle. On peut noter que la discussion sur les échelles temporelles est quasiment absente des différentes études menées 
(voir l'étude comparative d'Arhondisis et Brett, 2004). De la même façon, la validation des modèles, notamment empiriques, devrait faire partie des " bonnes pratiques » et l'obtention de critères quantitatifs de validation devrait être plus largement développée.

\section{Encadré 9.3}

\section{Exemple de modélisation pour étudier de la dynamique des masses d'eau océaniques}

\section{Pascal Boilly du Bois (IRSN)}

Les marqueurs radioactifs d'origine artificielle sont particulièrement intéressants pour les études de courantologie à long terme : leurs sources sont peu nombreuses et bien quantifiées, ils sont souvent solubles et peuvent êtres mesurés à des concentrations extrêmement faibles dans l'eau de mer (de l'ordre du $10000^{e}$ de la radioactivité naturelle). Les différentes sources connues peuvent ainsi être identifiées jusqu'à plusieurs centaines de kilomètres depuis le centre de la Manche et de la mer d'Irlande jusqu'à la sortie de la mer du Nord, par exemple (figure 9.7) il est alors possible d'apprécier le cheminement, la dilution des masses d'eaux et les temps de transit correspondants.

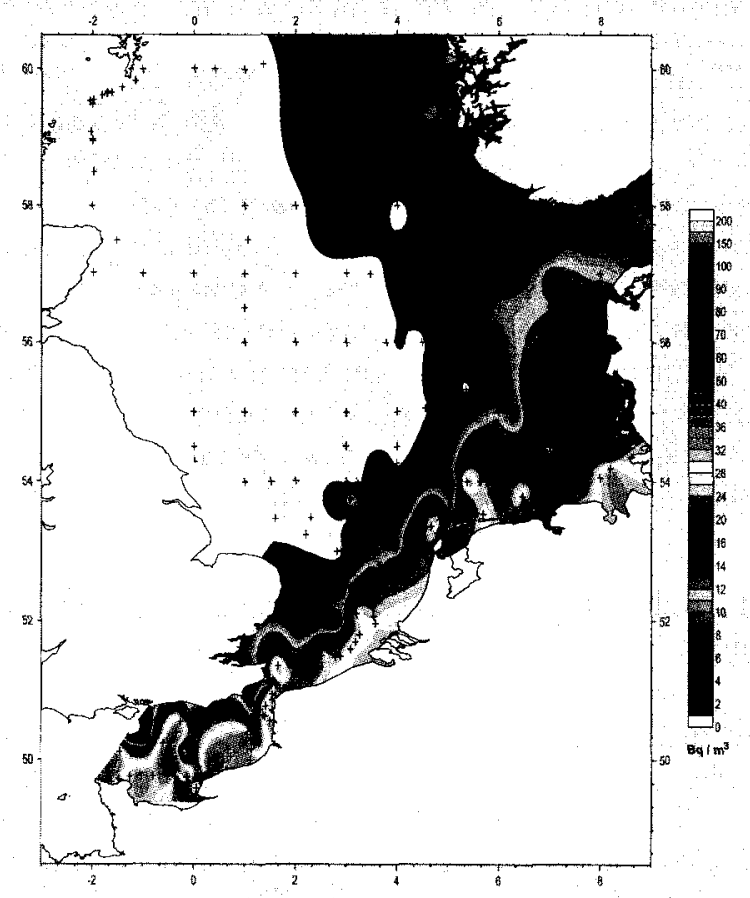

Figure 9.7

Antimoine 125 provenant des rejets de l'usine de La Hague dissous en mer du Nord en juillet 1988. 
et préciser les résultats des modèles hydrodynamiques de dispersion en mers macrotidales (Bailly du Bois et Dumas, 2005). Ces résultats sont transposables aux autres substances présentes dans leau de mer et permettent de simuler limpact à court, moyen et long terme diun rejet de substance dissoute sur le plateau continental du nord-avest de l'Europe. La connaissance du transport hydrodynamique est un préalable indispensable pour l'étude du comportement des substances ayant plus d'affinités pour les autres compartiments : sédiments et organismes vivants (Fiével et Plet, 2002). D'autres travaux montrent qu'ils peuvent être également utilisés dans l'étude des cinétiques de transfert de masses déeaux à plus grande échelle, en Arctique ef dans les mers environnantes (Strand ef al., 1996; Smith et al, 1997, Kershow et al., 2004).

Les travaux en cours utilisent les radiotraceurs pour préciser la dispersion de substances solubles dans l'eau de mer dans le champ proche d'un émissaire de rejet ainsi que pour caractériser le transport des sédiments déposés sur le fond. Les études à venir prendront en compte simultanément le devenir des radionucléides dans l'eau de mer, les sédiments et les espèces vivantes, à T'aide de modèles écologiques intégrés, validés par les mesures de radionucléides acquises in situ.

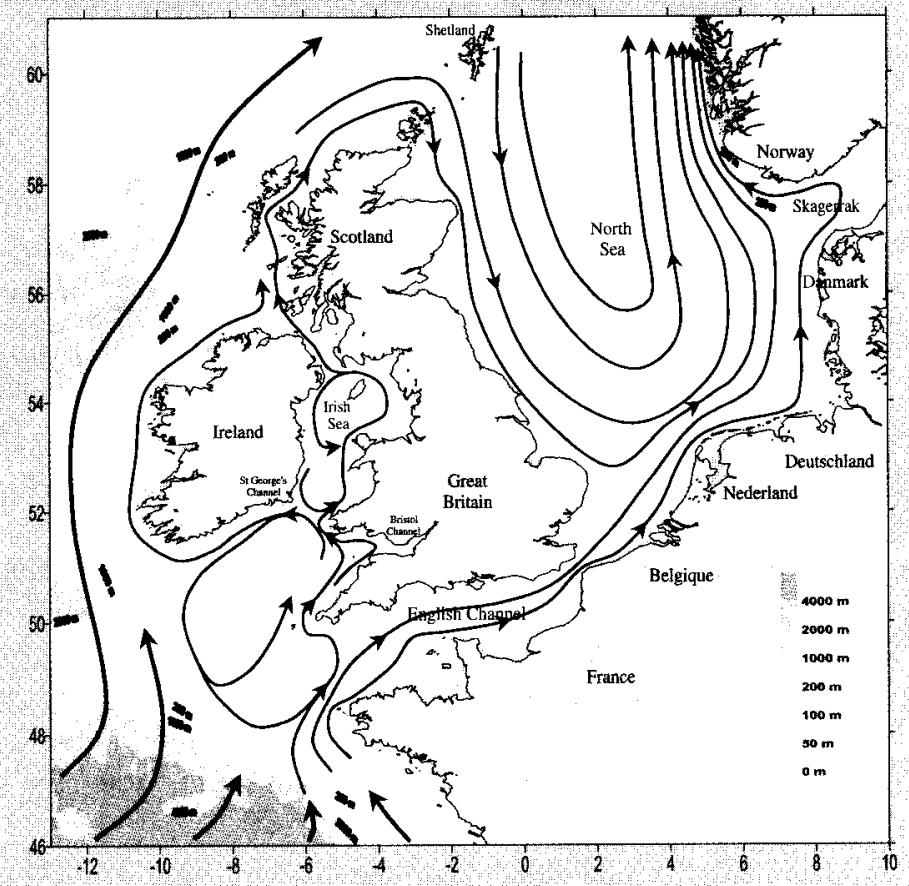

Figure 9.9

Circulation générale des masses deou sur le plateau continental du nord-ovest de lEurope. 
La distribution des radionucléides en mer du Nord résulte d'un mélange des radionucléides présents dans des eaux provenant de la Manche, de la Baltique, de l'Atlantique et de la mer d'Irlande. Un traitement mathématique permet de caractériser le pourcentage de chacune de ces contributions à léchelle de la mer du Nord, révélant ainsi une "photographie " de la répartition des masses d'eaux (figure 9.8).

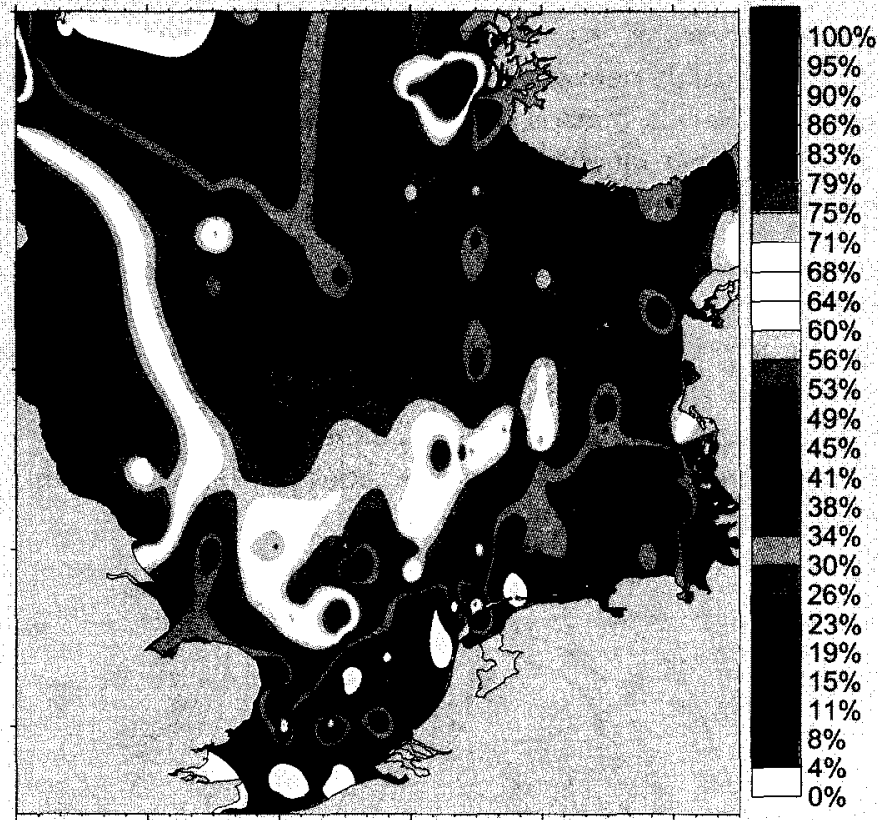

Figure 9.8

Répartition des eaux provenant de la mer d'flande en mer du Nord en 1988 (Bailly du Bois et al., 1993).

Grâce aux études sur le cheminement des radionucléides rejetés par l'usine de retraitement des combustibles usés de Cogema-la Hague effectuées entre 1987 et 1992, les caractéristiques du transit des eaux provenant de la Manche se dirigeant vers la mer du Nord ont été mises en évidence. Ces études montrent que les eaux mettent de 110 à 152 jours pour aller de la Hague au Pas-de-Calais et de 170 à 250 jours entre le Pas-de-Calais et le Skagerrak. La durée totale du transit entre le cap de la Hague et le Skagerrak serait de 300 à 370 jours selon les années. Le flux des eaux de la Manche vers la mer du Nord dans le Pas-de-Calais a été également estimé, de 97000 à $195000 \mathrm{~m}^{3} / \mathrm{s}$ de janvier à juillet 1988 .

Les radiotraceurs solubles ont permis de décrire la circulation à long terme des masses d'eau en Mer celtique, Manche et Mer du Nord (figure 9.9) (Bailly du Bois et al., 1993). L'association des travaux de radioécologie marine (IRSN) et de modélisation hydrodynamique (Ifremer) a permis de valider 


\section{Références bibliographiques}

André JC., Mégie G. et Schmidt-Lainé C. (2003). Échelles et changements d'échelles, problématiques et outils. In Caseau P. (ed). Études sur l'environnement : du territoire au confinent. RST $\mathrm{N}^{\circ} 15$, Académie des sciences, Tec \& Doc, Paris, p. 167-199.

Arhonditsis GB. et Brett MT. (2004). Evaluation of the current state of mechanistic aquatic biogeochemical modelling. Marine Ecology Progress Series, 271 : 13-26.

Bailly du Bois P., Guéguéniat P., Gandon R., Léon R. et Baron Y. (1993). Percentage contribution of inputs from the Atlantic, Irish Sea, English Channel and Baltic into the North Sea during 1988 : a tracer-based evaluation using artificial radionuclides. Netherlands Journal of Sea Research, 31 (1) : 1-17.

Bailly du Bois P. et Dumas F. (2005). Fast hydrodynamic model for of mediumand long-term dispersion in seawater in the English Channel and southern North Sea, qualitative and quantitative validation by radionuclide tracers. Ocean Modelling, $44 \mathrm{p}$.

Beaudoin N., Mary B. et Parnaudeau B. (1999). Impact of agricultural scenarios on nitrate pollution at the catchment scale. Présentation orale, 10 th Nitrogen Workshop, Copenhague (DK), 1999/08/23-26. Abstracts, 2 : IV-6.

Berner RA. (2003). The long-term carbon cycle, fossil fuels and atmospheric composition. Nature, 426 : 323-326.

Berner RA. (2004). The PhanerozoicCarbon Cycle. Oxford University Press. Une liste de modèles à LT sur ce sujet.

Billen G., Garnier J. et Hanset P. (1994). Modeling Phytoplankton Development in Whole Drainage Networks - the Riverstrahler Model Applied to the Seine River System. Hydrobiologia, 289 (1-3) : 119-137.

Bonnet MP. et al. (2000). Pelagic/Benthic Coupled 3D Modeling of Carbon and Nitrogen Cycling in the Gulf of Lions (NW Mediterranean Sea) : the Role of Sediments. Presented at Ocean Sciences Meeting, San Antonio, Texas.

Bricaud A. et al., (1999). Variations of light absorption by suspended particles with chlorophyll a concentration in oceanic (case 1) waters : Analysis and implications for bio-optical models (vol. 103, p. 31033, 1998). Journal of Geophysical Research-Oceans, 104 (C4) : 8025-8025.

Brisson N., Mary B., Ripoche D., Jeuffroy M., Ruget F., Nicoullaud B., Gate P., Devienne-Barret F., Antonioletti R., Durr C., Richard G., Beaudoin N., Recous S., Tayot X., Plenet D., Cellier P., Machet J., Meynard J. et Delécolle 
R. (1998). STICS : a generic model for the simulation of crops and their water and nitrogen balances. 1 - theory and parametrization applied to wheat and corn. Agronomie, $18: 311-346$.

Broecker WS. et Peng TH. (1982). Tracers in the Sea. Lamont-Doherty Geological Observatory, Palisades, N.Y. (épuisé).

Campbell J. et al., (2002). Comparison of algorithms for estimating ocean primary production from surface chlorophyll, temperature, and irradiance. Global Biogeochemical Cycles, 16 (3).

Cao M. et Wooward FI. (1998). Dynamic responses of terrestrial ecosystems carbon cycling to global climate change. Nature, 393 : 249-252.

Corman A. (1982). Modélisation mathématique du processus de nitrification dans les sols. Th. Doct. Ing. Université Claude Bernard lyon.

Davison W. et Motelica-Heino M. (2002). Influence of scale on trace metal dynamics in sediment and water. Geochimica et Cosmochimica Acta, 66 (15A): A172-A172.

Denis-Karafistan A. et al. (1998). Space and seasonal distributions of nitrates in the Mediterranean Sea derived from a variational inverse model. Deep-Sea Research Part I-Oceanographic Research Papers, 45 (2-3) : 387-408.

Evans GT. (2003). Defining misfit between biogeochemical models and data sets. Journal of Marine Systems, 40-41 : 49-54.

Even S. ef al. (1998). River ecosystem modelling : application of the PROSE model to the Seine river (France). Hydrobiologia, $374: 27-45$.

Feller et al. (2004). Les bases écologiques d'une fertilité durable des écosystèmes tropicaux culfivés et leurs perspectives. Acad. Agric. France.

Fiévet B. et Plet D. (2002). Estimating biological half-lives of radionuclides in marine compartments from environmental time-series measurements. Journal of Environmental Radioactivity, $65: 91-107$.

Flipo N. ef al. (2004). Biogeochemical modelling at the river scale : dynamics of phytoplankton and periphyton. Journal of Ecological Modelling, 176 : 333-347.

Gabrielle B., Mary B., Roche R., Smith P. et Gosse G. (2002). Simulation of carbon and nitrogen dynamics in arable soils : a comparison of approaches. Europen Journal of Agronomy, 18 (1-2) : 107-120.

Gomes E., Mignolet., Schott C., Brunstein D., Bornerand C., Ledoux E., Benoît M., Tournebize J., de Louvigny N., Ponsardin G. et Mary B. (1999). 
Dynamique agricole et pollutions nitriques diffuses: modélisation intégrée du tarnsfert des nitrates sur le bassin de la Seine. Rapport Piren Seine, 56 pages.

Hoteit I., Pham DT. et Blum J. (2003). A semi-evolutive filter with partially local correction basis for data assimilation in oceanography. Oceanologica acta, $26(5-6)$ : 511-524.

Justes E., Nicolardot B. et Mary B. (2001b). C and N mineralization of catch corp residues : measurements and evaluation of STICS mode. 11th Nitrogen Workshop, 9-12/09/2001, 113-114.

Karafistan A. et al. (2002). Space and time distributions of phosphate in the Mediterranean Sea. Deep-Sea Research Part I. Oceanographic Research Papers, $49(1): 67-82$.

Kershaw PJ., Heldal HE., Mork KA. et Rudjord AL. (2004). Variability in the supply, distribution and transport of the transient tracer ${ }^{99} \mathrm{Tc}$ in the NE Atlantic. Journal of Marine Systems, 44 (1-2) : 55-81.

Lancelot C. et Mathot S. (1985). Biochemical fractionation of primary production by phytoplankton coastal waters during short and long term incubations with 14C-bicarbonate, I, Mixed diatom population. Marine Biology, 86 : 219226.

Laugier M., Angot P. et Mortier L. (1996). Nested grid methods for an ocean model : A comparative study. International Journal for Numerical Methods in Fluids, 23 (11) : 1163-1195.

Laugier M., Mortier L. et Dekeyser I. (1994). A bidomain primitive equation model. Oceanologica Acta, 17 (4) : 355-367.

Lecourt D. (1999). Dictionnaire d'histoire et de philosophie des Sciences, p. 989-992, PUF.

Le Dimet FX., Navon IM. et Daescu DN. (2002). Second-order information in data assimilation. Monthly weather review, 130 (3) : 629-648.

Lions JL. (1997). Modélisation mathématique et environnement - Quelques remarques. In Blasco F. (ed). Nouvelles tendances en modélisation pour l'environnement. CNRS Programme Environnement, Vie et Sociétés/Elsevier, Paris, 79-90.

Lions JL., Temam R. et Wang S. (1992). Models for the coupled atmosphere and ocean. Comp Mech, $1: 1,3-120$.

Mills M. et al. (2004). Iron and phosphorus co-limit nitrogen fixation in the Eastern Tropical North Atlantic. Nature, 429 : 292-294. 
Morel A. et André JM. (1991). Pigment distribution and primary production in the western Mediterranean as derived and modeled from coastal zone color scanner observations. Journal of Geophysical Research, 96 : 12685-12698.

Motelica-Heino M. et Davison W. (2003). Trace metals dynamics in surface sediments investigated by DGT micro-scale measurements. Journal de Physique $N, 107: 899-902$.

O'Neill RV. (1988). Hierarchy Theory and Global Change. In Rosswall T. ef Woodmansee RG. (eds). Scales and Global Change. Scope 35. John Wiley \& Sons 29-46.

Prunet P., Minster JF., Ruiz-Pino D. et Dadou I. (1996a). Assimilation of surface data in a one-dimensional physical-biogeochimical model of the surface ocean, 1, Mechod and preliminary results. Global Biogeochem Cycles, $10: 111-138$.

Prunet P., Minster JF., Ruiz-Pino D. et Dadou I. (1996b). Assimilation of surface data in a one-dimensional physical-biogeochimical model of the surface ocean, 2, Adjusting a simple tropohic model to chlorophyll, temperature, nitrate and pCO2. Global biogeochem Cycles, 10 : 139-158.

Saugier B. (1997). Échanges de $\mathrm{CO}_{2}$ de l'écosystème à la biosphère. In Blasco F. (ed). Nouvelles tendances en modélisation pour l'environnement, CNRS Programme Environnement, Vie et Sociétés/ Elsevier, Paris, 31-46.

Schmidt-Lainé Cl. et Pavé A. (2002). Environnement : modélisation et modèles pour comprendre, agir et décider dans un contexte interdisciplinaire. Natures-Sciences-Société : Sciences pour l'ingénierie de l'environnement, 10 (s.1) : 5-25.

Schmidt-Lainé Cl. (1999). Échanges forêts-atmosphère : le cas du $\mathrm{CO}_{2}-$ Potentiel de recherche, questions scientifiques, protocole de Kyoto. Rapport à l'Académie des Sciences.

Schnebelen N. (2000). Analyse et modélisation de l'impact de la maîtrise des pratiques agricoles sur la pollution diffuse par les nitrates. Application à l'aquifère des calcaires de Beauce (site de Villamblain). Thèse de doctorat.

Servais P., Billen G. et Hascoët MC. (1987). Determination of the biodegradable fraction of dissolved organic matter in waters. Water research, 21 (4) : 445450.

Smith JN., Ellis KM., Kilius LR., Moran B., Molyak L. ef lvanov G. (1997). The transport of ${ }^{129} \mid$ and ${ }^{137} \mathrm{Cs}$ from European reprocessing plants through the Kara sea. Radioprotection, 32 (C2) : 97-103. 
Smith P. et al. Soil biota and global change at the ecosystem level : decribing soil biota in mathematical models. Global Change Biology, 4 : 773-784.

Soetaert K. et al. (2000). On the coupling of benthic and pelagic biogeochemical models. Earth-Science reviews, 51 : 173-201.

Soetaert K., Herman PMJ. et Middelburg JJ. (1996). A model of early diagenetic processes from the shelf to abyssal depths. Geochimica and Cosmochimica Acta, 60 (6) : 1019-1040.

Strand P., Sickel M., Aarkrog A., Bewers JM., Tsaturov Y. et Magnusson S. (1996). Radioactive contamination of the arctic Marine Environment. In Guéguéniat P., Germain P. et Métivier H. (eds). Radionuclides in the oceans, Inputs and Inventories. Les éditions de Physique, Les Ulis. p. 95-119.

Taillandier V. et al. (2004). Controlling boundary conditions with a fourdimensional variational data-assimilation method in a non-stratified open coastal model. Ocean Dynamics, 54 (2) : 284-298.

Tusseau MH. et al. (1997). 1D coupled physical-biological model of the northwestern Mediterranean Sea. Deep Sea Research II, 44 (3-4) : 851-880.

Tusseau-Vuillemin MH., Mortier L. et Herbaut C. (1998). Modeling nitrate fluxes in a open coastal environment (Gulf of Lions) : transport versus biogeochemical processes. Journal of Geophysical Research, 103 : 7693-7708.

Van Veen (1977). Nitrogen behaviour in soil; a computer simulation model. Ph. D. thesis, Wageningen, $164 \mathrm{p}$.

Viollier E. et al. (2003). Benthic biogeochemistry : state of the art technologies and guidelines for the future of in situ survey. Journal of Experimental Marine Biology and Ecology, 285 : 5-31.

De Wit CT. et al. (1978). Simulation of assimilation, respiration and transpiration of leaf surfaces. Pudoc, Wageningen.

Yool et Fasham MJR. (2001). An examination of the continental shelf pump in an open ocean general circulation model. Global Biogeochemical Cycles, $15(4): 831-844$. 

CHAPITRE 10
Mise en place et gestion de réseaux d'observation de longue durée 

La multitude des données indispensables à une étude approfondie des cycles biogéochimiques implique sans conteste la mise en place de réseaux d'observation permanents en vue de leur acquisition. De tels réseaux ont en effet plusieurs fonctions reconnues, comme par exemple :

- l'identification des évolutions des écosystèmes à différents pas de temps (court terme et surtout tendance à long terme);

- une fonction d'alerte à partir du suivi d'indicateurs pertinents de risque ;

- la calibration des modèles choisis.

$C^{\prime}$ est dans ce but qu'ont commencé à se développer divers réseaux nationaux sous la forme de GIS ou bien dans le cadre des ORE (observatoires de recherche en environnement) mis en place par le ministère de la Recherche en 2001.

Il s'agit maintenant d'aller plus loin. À cet effet, un point sera fait au cours de ce chapitre dans trois domaines :

- deux se rapportent à des écosystèmes naturels représentatifs de la surface de la planète : océans (10.1) et forêts (10.2);

- le troisième traite d'un problème très général, qui est celui des observatoires de la radioactivité (10.3). 



\section{SOUS-CHAPITRE 10.1}

\section{Observatoires océaniques et réseaux forestiers}

PATRICK RAIMBAULT ET JACQUES RANGER

I Observatoires océaniques : Les services d'observation du milieu marin (Patrick Raimbault)

L'emprise croissante de l'homme sur l'environnement pose le problème de sa vulnérabilité et des atteintes à son intégrité. C'est particulièrement vrai en ce qui concerne la préservation de la biosphère continentale et de l'environnement marin, ainsi que l'évolution climatique, trois domaines où la pression anthropique suscite des craintes à échéance de quelques dizaines $d$ 'années. Ces trois domaines sont étroitement couplés et leur étude doit être menée de front. Les études de séries temporelles à long terme de certains habitats aquatiques et terrestres ont significativement contribué aux sciences de l'Univers via la caractérisation des tendances climatiques fortes (exemple des pluies acides et de l'augmentation du $\mathrm{CO}_{2}$ atmosphérique).

On peut illustrer ce couplage avec le cycle du $\mathrm{CO}_{2}$ dont on soupçonne qu'il est en cours d'évolution rapide par suite des émissions artificielles pouvant entraîner des conséquences sur le climat. Mais on est encore loin d'avoir compris, 
par absence de mesures adéquates sur le long terme, le déséquilibre qui pourrait en découler. Étant insuffisamment alimentés par des données, les modèles d'évolution du milieu océanique du climat sont encore imparfaits. À l'aube d'une époque $d^{\prime}$ important changement climatique, il est essentiel de disposer de séries à long terme pour être en mesure d'interpréter les changements. La communauté ressent un important besoin de séries temporelles de données, que ce soit en point fixe ou sur des transects. Un ordre de grandeur de ces séries est de dix ans, afin de pouvoir isoler le signal anthropique, ou la variabilité interannuelle du signal saisonnier.

Observer la nature pour en déduire les lois de son fonctionnement fait partie intrinsèquement de la démarche scientifique et ceci n'est pas nouveau.

L'observation implique une attitude volontaire dont la fréquence et la régularité vont dépendre de l'objet de l'étude comme de l'espace de temps supposé avant de déceler un changement attendu. Quand celui-ci est de très faible amplitude à une échelle décennale comparativement à sa variabilité à de plus faibles échelles temporelles, ou que les contraintes du local masquent un signal plus global, précision, régularité et pérennité des mesures sont des impératifs. Or, les contraintes du milieu et les problèmes logistiques du travail en mer rendent l'obtention de ces qualités beaucoup plus difficiles qu'en milieu continental. Aussi, à la différence par exemple de la météorologie, des séries régulières sur le long terme de paramètres physicochimiques n'étaient pas fréquentes dans le milieu marin jusqu'à la mise en place par l'Insu (Institut national des sciences de l'Univers), par la fédération de séries existantes ou la création de nouvelles, de sites d'observation océaniques dans les années 1980 (Kerfix, Oiso, Dyfamed) et un peu plus tard dans le domaine côtier (Somlit créé en 1996).

Dans ce cadre, les objectifs de l'observation sont triples : connaître le passé, observer le présent et prévoir le futur.

Connaître le passé, c'est savoir comment ont évolué certaines caractéristiques de l'océan dans un passé proche et en particulier depuis le début de l'ère industrielle. Cela implique la recherche d'archives et la mise en perspective des données qu'elles contiennent.

Observer le présent, c'est mener une action volontaire, répétitive d'observation de l'évolution des caractéristiques du milieu marin par des mesures systématiques à des emplacements fixes considérés comme représentatifs.

Prévoir l'avenir est le but in fine. Discerner les différentes échelles de temps des phénomènes, dégager le local du général, les petites variations qui s'accumulant peuvent modifier les milieux naturels sur le long terme. 
Une meilleure compréhension du cycle du carbone océanique et une meilleure connaissance de l'impact de l'océan vis-à-vis d'une probable augmentation de l'effet de serre nécessitent que l'on observe et que l'on comprenne les variations saisonnières à moyenne échelle de la pression partielle de dioxyde carbone $\left(\mathrm{pCO}_{2}\right)$ dans les eaux de surface de l'océan mondial. Les programmes nordaméricains HOT (Hawai Ocean Time-Series dans le Pacifique Nord subtropical) et BATS (Bermuda Atlantic Time-series Study dans la mer des Sargasses) ont permis l'implantation et le maintien de longue date de stations échantillonnées régulièrement pour le suivi de paramètres physiques et chimiques.

En France également, la nécessité de tels dispositifs s'est imposée très tôt aux océanographes biogéochimistes et certains systèmes d'observation sont fonctionnels depuis plusieurs années. Dès le début des années 1980, un effort a été effectué en ce sens avec le soutien de l'Insu-CNRS pour acquérir de séries de données notamment dans l'océan austral - opération Minerve, et la composante française de JGOFS (Proof) a permis la création de la station Kerfix au large des îles Kerguelen, du suivi Oiso et récemment de l'Ore Caraus dans l'océan Indien austral. Ces services d'observation ont bénéficié du développement de mesures automatisées et peuvent être réalisés grâce aux transits récurrents des navires assurant la logistique pour les îles australes et l'Antarctique). Un site équivalent aux stations HOT et BATS existe depuis 1980 en Méditerranée nord-occidentale (Dyfamed). Ces stations ont pour but d'améliorer la compréhension du cycle global du carbone et des éléments associés, et d'étudier la variabilité océanique sur des échelles de temps allant du jour à la décennie.

Il est à noter que ces sites d'observations à long terme, essentiellement de type océanique, sont fréquemment le support d'opérations scientifiques plus ponctuelles auxquelles ils apportent la composante variabilité interannuelle.

Ces services d'observation fonctionnent correctement, bien que le problème de leur inscription dans la durée se pose à tous leurs responsables; la difficulté étant de pouvoir disposer de personnel à plein temps pour en assurer la régularité du suivi. Il est impératif que les services d'observation soient dotés de moyens humains nécessaires pour pérenniser la continuité des observations acquises sur plus de dix ans.

Dans le domaine côtier la situation est sans doute moins claire. L'interférence entre une fluctuation naturelle élevée et l'effet des facteurs anthropiques complique notablement l'interprétation de la variabilité basse fréquence. La déconvolution des variations climatiques à méso-échelle et des changements globaux, en partie dus aux activités humaines, est un préalable à l'interprétation des réseaux d'observation côtiers. La zone côtière constitue une étape dans le devenir de la matière entre le domaine continental et le domaine océanique. Les variations spatiales et temporelles peuvent être importantes et très dépendantes 
des processus physiques et hydrodynamiques locaux. Des mesures océanographiques très fréquentes sont donc impératives en milieu côtier pour quantifier les processus côtiers et de paramétrer leurs forçages.

Un dispositif d'observation du milieu littoral (Somlit) a été mis en place en 1996 par l'Insu au sein du Réseau national des stations marines (RNSM) qui peut répondre en partie à ces besoins. Une mobilisation plus large des organismes a été engagée par la mise en place des Ore (observatoires régionaux de l'environnement) mais qui n'a pas pour le moment évolué vers la mise en place de systèmes pérennes.

Actuellement, les contraintes sur les moyens humains et logistiques font qu'il est impossible de procéder à un suivi à très haute fréquence. En effet, ces sites d'observation côtiers ne sont pas équipés de systèmes de mesures autonomes, et la plupart du temps ces études reposent sur des prélèvements hebdomadaires et sont très coûteuses en temps et personnels.

De plus, la mise en place de la surveillance induite par la directive cadre sur l'eau (DCE) nécessite une redéfinition des réseaux de surveillance existant dans le domaine côtier. La classification des états écologiques repose sur l'étar de qualité, notamment biologique et physicochimique. La qualité biologique est ainsi caractérisée par la composition, l'abondance et la biomasse du phytoplancton. La qualité physicochimique est évaluée par la transparence, la température, la salinité la concentration en oxygène dissous et en nutriments. La stratégie de surveillance des réseaux hydrologiques doit donc évoluer à la fois en termes de fréquence de mesures, de nombre de points de prélèvements et de type de paramètres à analyser. Et il est certainement indispensable de disposer de séries de mesures fines, notamment dans une problématique d'alarme.

Ces séries ne peuvent être obtenues qu'avec l'aide d'instrumentations in situ adaptées au milieu côtier. La mise en place de stations autonomes multiinstrumentées doit être intensifiée ainsi que le développement de capteurs autonomes in situ pour des paramètres chimiques (analyseur de sels nutritifs) et biologiques (cytomètre en flux, spectrofluorimètre par exemple).

En complément aux mesures in situ, les informations fournies par les satellites représentent des données océaniques d'un très grand intérêt. Elles permettent d'établir des séries temporelles synoptiques à un rythme serré et renferment une information très précise sur les déplacements des masses d'eau, les remontées d'eau profonde et, associées aux données d'altimétrie et de température de surface de l'océan, elles procurent une vision assez détaillée de l'océan à mésoéchelle. 
Il est important de soutenir les travaux qui sont en cours afin de combiner les données de satellites différents (infrarouge, altimétrie, couleur de l'eau) pour constituer des séries longues, sur lesquelles un signal anthropogénique pourrait être détecté.

II est important que les services d'observation continuent à être situés près d'un laboratoire de recherche afin d'en maintenir le dynamisme, d'en assurer la qualité des données, et favoriser l'utilisation des observations pour répondre à des questions de recherche. II est urgent que les services d'observation soient dotés de moyens humains nécessaires pour pérenniser la continuité des observations qui doivent être acquises sur le long terme et que les mesures en continu autonomes viennent rapidement améliorer la stratégie des suivis à long terme, notamment dans les domaines côtier et littoral. 


\section{Réseaux forestiers (Jacques Ranger)}

Environ trente-cinq réseaux forestiers ont été recensés dans le document Les chemins de l'information forestière (publication du GIP-Ecofor, 2000). Ces réseaux $y$ sont regroupés par thème : les réseaux produisant les statistiques forestières avec en particulier l'Inventaire forestier national créé en 1958 et le Service central des études statistiques des ministères en charge de l'Agriculture, la Forêt et la Pêche créé en 1961, les réseaux de surveillance de la santé des forêts, les réseaux de surveillance des risques naturels, les réseaux de suivi du fonctionnement des écosystèmes forestiers, et les réseaux de conservation des milieux naturels et du patrimoine.

Si tous ces réseaux intéressent de près ou de loin les cycles biogéochimiques, cette présentation se focalisera sur un petit nombre, plus particulièrement concernés.

L'origine des réseaux de surveillance des forêts en Europe réside dans la crise majeure du dépérissement des forêts au cours des années 1980, souvent attribuée de façon simpliste aux seuls apports atmosphériques acides. Les préoccupations se sont ensuite déplacées au cours des années 1990 vers la question des changements climatiques. Les fonctions de ces réseaux sont la surveillance des écosystèmes et l'alerte, et, de façon moins directe, l'amélioration des connaissances.

La réglementation communautaire européenne des années 1980 a conduit les États membres à mettre en place un réseau d'observation, le Réseau européen de suivi des dommages forestiers dit réseau européen. Ce réseau, installé à partir de 1988, est de type systématique puisque les 540 placettes retenues sont distribuées aux nœuds d'une maille de 16 par $16 \mathrm{~km}$. Cette maille a été choisie pour intégrer des points d'observations préalablement mis en place dans des réseaux nationaux (cas entre autres du réseau 16 par $1 \mathrm{~km}$ dit " réseau bleu", des zones montagneuses françaises). Les observations concernent les données écologiques descriptives (sols, végétation spontanée et peuplements forestiers) et le statut sanitaire des peuplements observé annuellement (défoliation, coloration, symptômes de dommages biotiques et abiotiques...). Les résultats sont publiés dans les cahiers du département Santé des forêts (La santé des forêts) de ministère en charge de l'Agriculture (publication annuelle) et dans les rapports du PIC Forêts.

En 1992, suite à la Conférence ministérielle de Strasbourg, un règlement de I'Union européenne décrète la mise en place d'un réseau plus intensif (dit " de niveau \|\| , venant en appui du précédent. La partie française de ce réseau est 
réalisée dans Réseau national de suivi des écosystèmes forestiers dit "réseau Renécofor " sous la responsabilité de l'Office national des Forêts. II s'agit d'un réseau non systématique comportant 102 placettes permanentes d'observation, représentant les grands types de forêts rencontrés sur le territoire métropolitain et prévues pour être suivies pendant au moins trente ans. Trois niveaux d'approche sont réalisés : le niveau 1 examine la végétation (arbres et végétation spontanée) et les sols, le niveau 2 prend en compte les apports atmosphériques (27 placettes) et le niveau 3 observe en plus les solutions du sol (17 placettes du niveau 2). Les données relatives au réseau Renécofor font l'objet d'un programme assurance qualité spécifique pour chaque domaine étudié, elles sont stockées dans une base de données centralisée et elles sont publiées par l'Office national des forêts dans des dossiers thématiques disponibles auprès du responsable du réseau (E. Ulrich, Boulevard de Constance, 77300 Fontainebleau). Une présentation de ce réseau et une sélection des principaux résultats sont accessibles sur le site de l'ONF (www.onf.fr/pro/renecofor/index.htm).

La recherche accompagne ce mouvement par la création de sites-ateliers fortement instrumentés et suivis sur le moyen terme. La promotion et la structuration des recherches sur le fonctionnement biogéochimique des écosystèmes forestiers a été améliorée par la création en 1993 d'un groupement d'intérêt public, le GIP-Ecofor. La mise en réseau de ces sites de recherche, dont le nombre est inférieur à 10, a été formalisée en 2002 dans le cadre de la création de l'Observatoire de recherche en environnement F-ORE-T, coordonné par le GIPEcofor. Ces sites sont des laboratoires d'écologie in situ, visant à identifier les mécanismes du fonctionnement biogéochimique et à quantifier les flux d'eau et d'éléments (incluant le carbone) entrants, sortants et internes à l'écosystème. Les documents relatifs à ce réseau sont disponibles auprès des organismes de recherche et auprès du GIP-Ecofor (6, rue du Gal Clergerie, 75116 Paris).

Ajoutons l'existence d'un réseau de sites expérimentaux d'épandage des déchets en forêt, coordonné par l'Inra. Il est destiné à tester les contraintes biologiques et chimiques pour les sols et les eaux de surface, qui résulteraient de cette pratique actuellement interdite. Les documents sont disponibles à l'unité de recherche forestière du centre Inra de Bordeaux (Pierroton, 33610 Cestas).

La connexion des réseaux forestiers français, voire européens est réalisée dans les projets de généralisation, voire de spatialisation des données locales, soit pour quantifier des flux, soit pour valider les modèles et développer des simulateurs simplifiés. Les modèles à base mécaniste sont développés dans l'ORE F-ORE-T et leur validation après simplification sera réalisée à partir des bases de données des réseaux de type OOE (Observatoire opérationnel en environnement) tel le Renécofor. 
Un observatoire de la qualité des sols agricoles est créé en 2003, le Réseau de mesures de la qualité des sols (RMQS). Ce réseau de maille systématique est pris en charge par un groupement d'intérêt scientifique regroupant les ministères en charge de l'Agriculture et de l'Environnement, l'Ifen, l'Ademe et l'Inra. Ses objectifs concernent la protection de l'environnement, les risques de contamination de la chaîne alimentaire et l'adaptation de l'agriculture aux nouveaux règlements et contraintes économiques. Il comporte environ 1500 points situés sur la même grille que le réseau européen de surveillance de la santé des forêts (500 points) qui présente également un volet " sols ». Ces deux réseaux devraient être plus particulièrement associés au premier ré-échantillonnage (pas de sept à huit ans prévu pour le RMQS). Les documents relatifs à ce réseau sont disponibles à l'Unité Infosol (Inra Centre d'Orléans, 45000 Olivet).

\section{Références bibliographiques}

GIP-Ecofor (2004). Rapport d'activité du réseau ORE F-ORE-T.

Département de la Santé des Forêts (2004). Les Cahiers du DSF, 1-2003/2004 (La Santé des Forêts (France) en 2002), Min. Agri. Alim. Pêche Aff. Rur. (DGFAR), Paris, 91 p. + ann.

Renaud JP. (2004). Les résultats 2002 du réseau européen de suivi des dommages forestiers. Les Cahiers du DSF, 1-2003/2004 (La Santé des Forêts (France) en 2002), Min. Agri. Alim. Pêche Aff. Rur. (DGFAR) Paris, p. 46-60.

Office national des forêts, département des Recherches techniques (1996) : Notice de présentation du Réseau national de suivi à long terme des écosystèmes forestiers. ISBN 2- 84207-021-6, 38 p.

ou rapports PIC Forêts (http://www.icp-forests.org/RepEx.htm;

http://www.icp-forests.org/RepTecl.htm). 


\section{SOUS-CHAPITRE 10.2}

\section{Observatoire permanent de la radioactivité}

EVELYNE BARKER, PATRICK BOUISSET, PIERRE GERMAIN, OLIVIER MASSON ET HENRI MÉTIVIER

Des réseaux de surveillance à caractère réglementaire, mis en œuvre par les industries du nucléaire et les organismes chargés du contrôle de la radioactivité dans l'environnement, existent dans de nombreux pays (la plupart des pays européens, le Canada, les États-Unis, le Japon. . .).

En France, I'IRSN a en charge, outre ces aspects de surveillance radiologique du territoire, un observatoire de la radioactivité. Ce système d'observation, appelé Opera ${ }^{1}$, concerne l'atmosphère et les différents compartiments de la biosphère (milieux terrestres, fluviaux et marins).

Cette mission d'observation a été initiée en 1959 pour répondre à des préoccupations sanitaires, au moment où les essais nucléaires dans l'atmosphère battaient leur plein à des fins militaires.

Développé à l'origine pour le milieu atmosphérique, l'observatoire couvre aussi le littoral des côtes françaises (sédiments, bio-accumulateurs algues et

${ }^{1}$ Observatoire permanent de la radioactivité de l'environnement. 
moules) depuis 1983, le milieu terrestre (sols, végétaux spontanés, denrées agricoles) depuis 1993, ef le domaine fluvial (eaux, matières en suspension) depuis 1998. L'observatoire s'appuie aujourd'hui sur un réseau de plus de trente-cinq stations et fonctionne grâce à de nombreuses collaborations logistiques et scientifiques (universités, instituts) (figure 10.2-1).

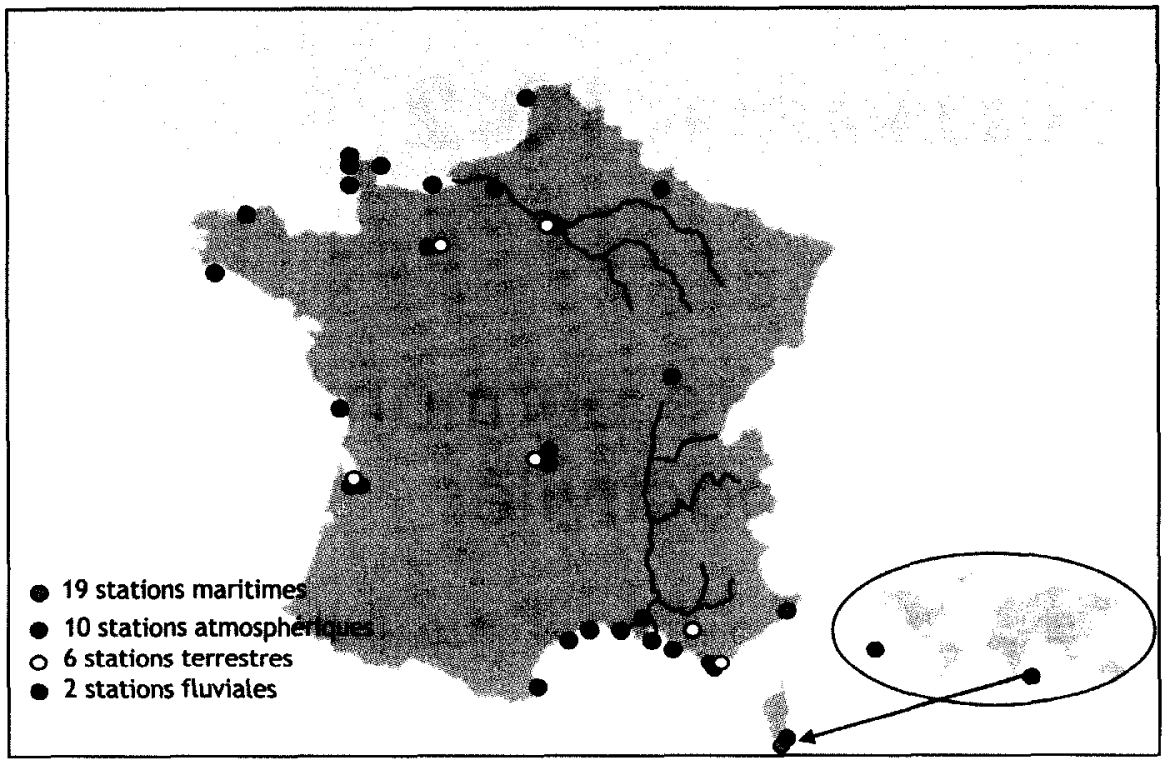

Figure 10.2-1

Localisation des stations de l'Observatoire permanent de la radioactivité de l'environnement.

À ce jour, l'objectif principal de cet observatoire est la connaissance des niveaux d'activité et des mécanismes responsables des évolutions temporelles et spatiales des radionucléides naturels et artificiels présents dans l'environnement à l'état de traces, voire d'ultratraces. Les performances des moyens mis en œuvre permettent notamment de quantifier la radioactivité artificielle très en deçà des seuils accessibles aux autres réseaux existants. Afin d'atteindre cet objectif, tous les maillons d'une chaîne allant de la collecte à la mesure en passant par les traitements sont dimensionnés pour concentrer les radionucléides. Ainsi, jusqu'à $100000 \mathrm{~m}^{3} \mathrm{~d}^{\prime}$ air, plusieurs milliers de litres d'eau et plusieurs kilogrammes d'organismes vivants sont régulièrement prélevés.

De par cette capacité, les fluctuations des niveaux d'activité dans l'environnement peuvent être mises en parallèle avec la variabilité des phénomènes naturels liés aux cycles biologiques des espèces collectées, à la géochimie ou à la climatologie des sites étudiés. Les données obtenues dans le cadre de ce programme pluriannuel pérenne ont permis la constitution de séries chronologiques 
indispensables à de nombreuses études scientifiques. À ce titre, Opera multiplie les échanges avec d'autres systèmes d'observation et observatoires de recherche en environnement mis en place avec le soutien de l'Insu' ${ }^{2}$.

Outre la communication scientifique, les résultats obtenus dans le cadre d'Opera sont régulièrement mis à la disposition du public via le site Internet de l'IRSN (www.irsn.org/opera).

Sont donnés ci-après quelques exemples clés relatifs aux différents compartiments étudiés dans le cadre d'Opera.

\section{1 Étude de l'atmosphère}

Deux exemples seront pris à ce sujet :

- celui de la série temporelle du ${ }^{137}$ Cs dans les aérosols;

- celui de la connaissance des mouvements des mouvements atmosphériques planétaires.

\section{Exemple de la série temporelle du ${ }^{137}$ Cs dans les aérosols (figure 10.2-2)}

Jusque dans les années 1980 , la contamination de l'air en ${ }^{137} \mathrm{C}$ s provenait des essais nucléaires atmosphériques, conduisant à des concentrations dépassant 1000 microbecquerels par mètre cube $\mathrm{d}^{\prime}$ air $\left(\mu \mathrm{Bq} \cdot \mathrm{m}^{-3}\right)$ dans les années 1965. L'arrêt des essais, associé à la décroissance radioactive du ${ }^{137} \mathrm{Cs}^{31}$ et aux mouvements des masses d'air, ont conduit à des valeurs de l'ordre de $1 \mu \mathrm{Bq} \cdot \mathrm{m}^{-3}$ d'air avant l'accident de Tchernobyl (26 avril 1986). Cet accident a entraîné une contamination forte mais brève de l'atmosphère en France.

Dès les années 1993-1994, les prélèvements sont caractérisés par des niveaux d'activité équivalents à ceux précédant l'accident. La diminution s'est poursuivie jusqu'à la fin des années 1990 pour se stabiliser ces dernières années à des valeurs de l'ordre de $0,3 \mu \mathrm{Bq} \cdot \mathrm{m}^{-3}$.

Cette série chronologique a permis de mettre en évidence un autre évènement survenu fin mai 1998 à la suite de l'incinération incidentelle d'une source

\footnotetext{
${ }^{2}$ Institut national des sciences de l'Univers.

${ }^{3}$ La concentration en ${ }^{137} \mathrm{Cs}$ diminue de moitié en trente ans (période de décroissance radioactive).
} 


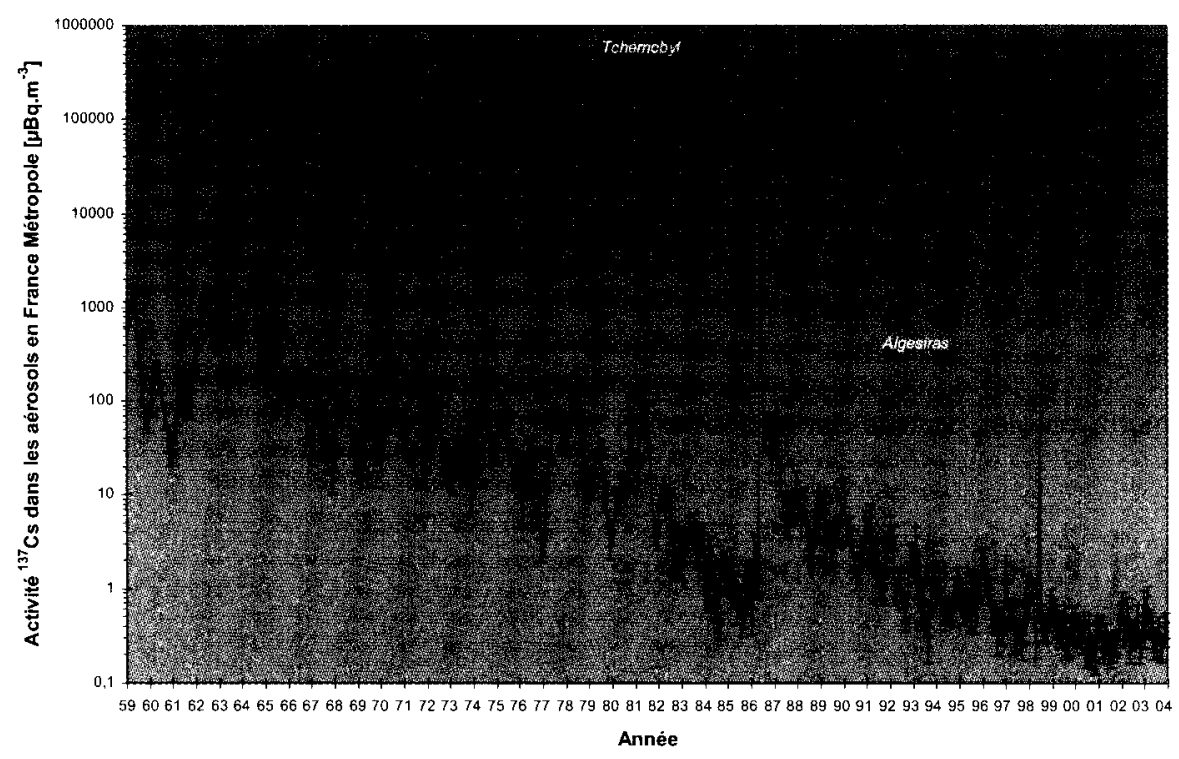

Figure 10.2-2

Activité volumique du ${ }^{137} \mathrm{Cs}$ dans les aérosols en France métropolitaine (Bouisset, 2004).

radioactive de ${ }^{137} \mathrm{Cs}$ dans une aciérie du sud de l'Espagne à Algésiras. Une contamination de l'atmosphère en ${ }^{137}$ Cs a été enregistrée dans l'Europe du Sud par les systèmes de surveillance des différents pays. Avec leurs meilleures sensibilités, les stations de l'observatoire ont permis de montrer l'impact significatif de l'incident dans l'atmosphère sur l'ensemble du territoire à l'exception du NordOuest de la France. En quelques jours, les niveaux de radioactivité sont revenus à la normale et l'évènement $n^{\prime}$ a pas laissé de traces observables dans les autres compartiments de la biosphère.

À une échelle plus fine, ces séries temporelles permettent la caractérisation des mécanismes intervenant de façon permanente ou évènementielle, comme par exemple la remise en suspension de particules antérieurement déposées et à l'origine du maintien du bruit de fond actuel de $0,3 \mu \mathrm{Bq} \cdot \mathrm{m}^{-3}$ ou les dépôts par temps de pluie qui momentanément diminuent ce bruit de fond.

Ces observations ont débouché sur la mise en œuvre de programmes de recherche spécifiques en collaboration avec des universités, par exemple sur les mécanismes d'incorporation des radionucléides dans les pluies, l'influence de l'origine océanique ou continentale des masses d'air ou sur les apports évènementiels (poussières sahariennes, grêle, ...). Parmi les questions clés dans le compartiment atmosphérique, restent en suspens notamment l'étude de la répartition des niveaux d'activité dans le plan vertical, la contribution respective 
des remises en suspension locale et distante ou encore les dépôts occultes sur les sites soumis à des brouillards ou immergés dans les nuages.

\section{Mouvements atmosphériques planétaires}

Les essais aériens d'engins nucléaires ont essentiellement été faits dans l'hémisphère Nord. Ils ont injecté dans la haute atmosphère des débris d'armes (uranium et plutonium) et des produits de fission et des actinides lourds créés au moment de l'explosion. Les aérosols qui s'ensuivent descendent par gravité et sont transportés avec les mouvements d'air aux altitudes plus faibles. Les mouvements des masses d'air dépendent des saisons et le retour sur terre dépend également de la pluviosité régionale. Le temps moyen de résidence dans la basse atmosphère est de trois à douze mois dans les régions polaires et de huit à vingt-quatre mois dans les régions équatoriales. L'analyse des radionucléides a permis d'avancer dans la description des mouvements d'air dans les différentes parties atmosphériques, comme le montre la figure 10.2-3 tirée du rapport UNSCEAR 2000 mais aussi dans la quantification des échanges entre les strates atmosphériques, expliqués dans le même rapport. On voit sur la figure que les aérosols injectés dans l'atmosphère par les explosions descendent par gravité aux altitudes les plus hautes puis sont transportées par les mouvements classiques d'air dans les couches les plus basses. La diffusion cause des migrations irrégulières des masses d'air dans la couche basse de la stratosphère et la couche haute de la troposphère (diffusion de Eddy). Le flux d'air circulaire de la troposphère aux plus basses altitudes, appelé circulation dans les cellules de Hadley, montre que ces cellules croissent ou décroissent en taille et se déplacent selon la latitude avec les saisons.

Des valeurs chiffrées ont pu être associées à ces mouvements. Par exemple, pour les mois de mars, avril et mai, d'une part et septembre, octobre et novembre d'autre part, les temps de résidence moyen des aérosols dans la basse stratosphère sont de trois à douze mois dans les régions polaires et de huit à vingt-quatre mois dans les régions équatoriales. Les temps d'épuration les plus rapides sont observés au printemps. D'autres valeurs numériques ont été données pour les autres compartiments. Sur la base de ces données des modèles empiriques ont été développés.

L'importante dispersion de débris en 1962 a également permis de montrer l'absence de dispersion de ceux-ci dans l'hémisphère Sud à partir des injections faites dans l'hémisphère Nord. Enfin le suivi des concentrations à très basse altitude après les essais, puis après l'accident de Tchernobyl a permis de montrer l'importance de la re-suspension des particules du sol dans la concentration en particules de l'atmosphère (voir figure 10.2-2). 


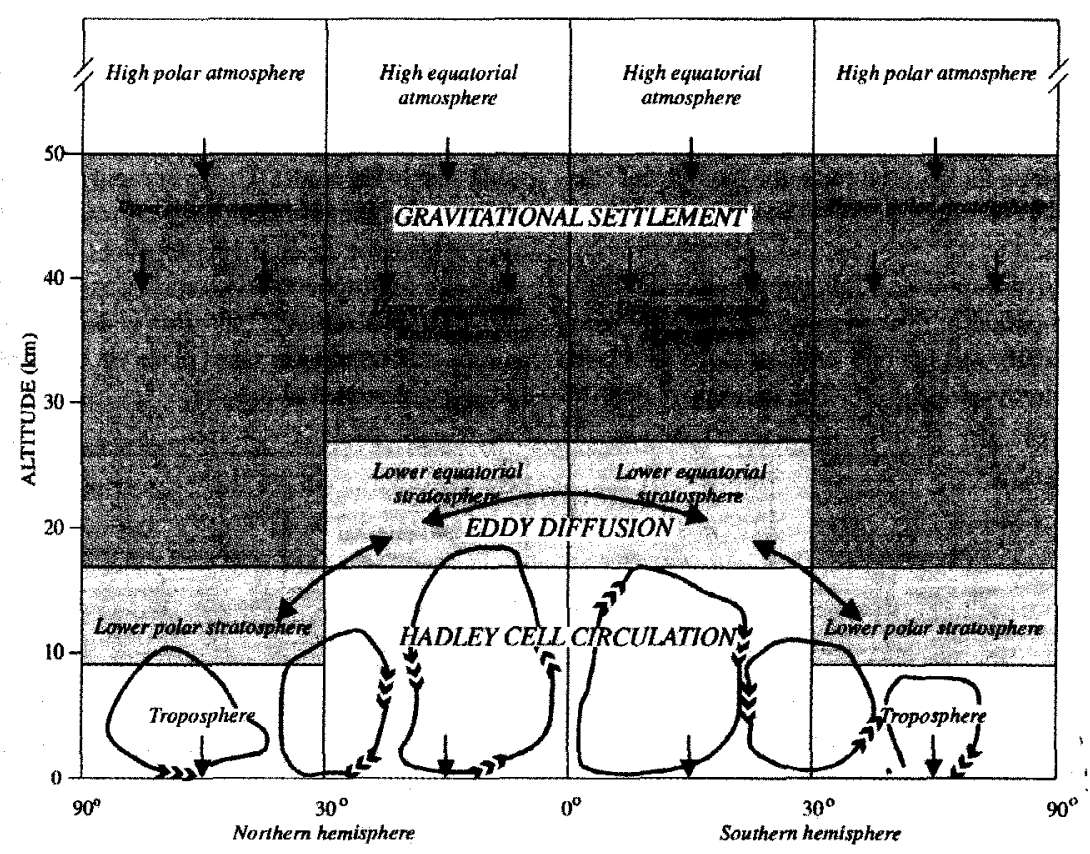

Figure 10.2-3

Régions atmosphériques et procédés de transport atmosphérique prédominants (UNSCEAR, 2000).

Dans ce qui suit, on montre comment une observation permanente au sein d'un observatoire de la radioactivité de l'air a permis d'expliquer une part de ces phénomènes de redistribution, en particulier la différence saisonnière entre une contamination provenant de la haute atmosphère et d'une contamination provenant $d^{\prime}$ une remise en suspension d'une contamination terrestre.

\section{2 | Étude du milieu terrestre}

L'examen des données de la période 1993-2004 a permis d'acquérir des connaissances concernant les niveaux d'activité et le comportement du ${ }^{137} \mathrm{Cs}$ dans les sols, les végétaux et les produits d'origine animale dans trois régions françaises (Normandie, région Centre et Sud-est).

Les prélèvements de sols et de produits agricoles (légumes, herbe, lait, fromage, viande) s'inscrivent dans une logique d'étude des transferts. Sont étudiées notamment les chaines sol-herbe-lait-fromage et sol-herbe-viande. 
On observe une diminution généralisée des activités massiques des sols en surface, des végétaux et des produits d'origine animale (figure 10.2-4), avec des périodes apparentes de décroissance, variables selon les compartiments et la localisation des prélèvements, de l'ordre d'une dizaine d'années.

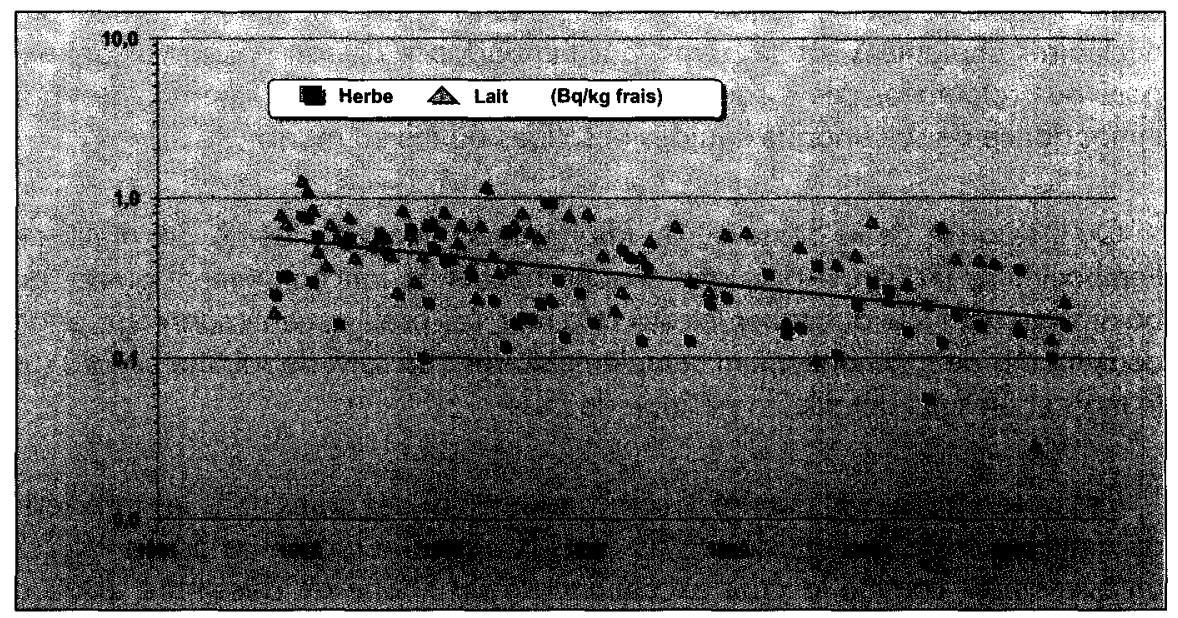

Figure 10.2-4

Évolution de lactivité volumique en ${ }^{137} \mathrm{Cs}$ de l'herbe et du lait de vache.

Le suivi au cours du temps des facteurs de transfert permet de mettre en évidence la diminution du transfert racinaire qui traduit d'une part la décroissance de l'activité superficielle du sol et, d'autre part, la diminution de la biodisponibilité du césium pour les végétaux.

Dans la couche de surface des sols, on observe une grande disparité des concentrations en ${ }^{137} \mathrm{Cs}$, en raison de l'hétérogénéité des dépôts atmosphériques (retombées des essais atmosphériques et de l'accident de Tchernobyl). Ces différences peuvent ensuite être amplifiées ou réduites par le biais des processus d'accumulation ou de décroissance, dont l'intensité varie avec les caractéristiques du sol et son utilisation. Ainsi, plutôt que d'acquérir quantités de mesures ponctuelles sur différentes stations, les stratégies s'orientent vers l'acquisition des chroniques de mesures et l'étude approfondie des chaînes de transfert. À cet effet, certaines des stations terrestres d'Opera sont associées à des zones ateliers ${ }^{4}$, sur lesquelles des recherches plus fines sont réalisées.

\footnotetext{
${ }^{4}$ Périmètre d'étude in situ, sur un thème ciblé, pour une durée limitée.
} 


\section{3 | Étude du milieu fluvial}

Deux bassins versants aux caractéristiques bien différenciées font l'objet d'un suivi dans le cadre d'Opera : la Seine depuis 2000 et le Rhône depuis 2003. Le choix de cette deuxième station a été dicté à la fois par le nombre d'installations nucléaires implantées sur ses rives, par l'importance de son bassin versant exposé aux retombées des essais nucléaires et de l'accident de Tchernobyl et par l'importance de ses apports liquides et solides au milieu marin.

Qu'il s'agisse de la Seine ou du Rhône, la station est située à proximité de l'embouchure (Roven, Arles) afin d'appréhender le fonctionnement global du bassin versant et de quantifier les flux à la mer. Les prélèvements sont régis selon deux modes de fonctionnement complémentaires : un suivi de routine intégré sur le mois et un suivi détaillé en période de crue.

Ces observations ont montré le rôle fondamental des crues dans les transferts de matières et de radionucléides associés. Comme le montre la figure 10.2-5 un évènement comme la crue exceptionnelle du Rhône de décembre 2003 a représenté à lui seul plus de $70 \%$ du flux annuel véhiculé sous forme particulaire et sous forme dissoute.

Pour les situations d'étiage, les flux particulaires et dissous sont du même ordre de grandeur.

Ces stations constituent des outils exceptionnels en raison de l'originalité des solutions techniques employées et ont été proposées comme plates-formes instrumentées à disposition de la communauté scientifique pour des recherches sur divers composés (sels nutritifs, micropolluants...).

\section{4 | Étude du milieu marin}

Débutée dès 1983 en Méditerranée, la couverture géographique du volet marin Opera concerne l'ensemble de la façade maritime française (Manche, Atlantique, Méditerranée) par l'intermédiaire de 19 stations de collecte de bioindicateurs (moules, algues, poissons) et de sédiments.

Le ruthénium 106 (période de décroissance radioactive d'un an) est un élément marqueur des rejets des centres de retraitement du combustible (La Hague, 


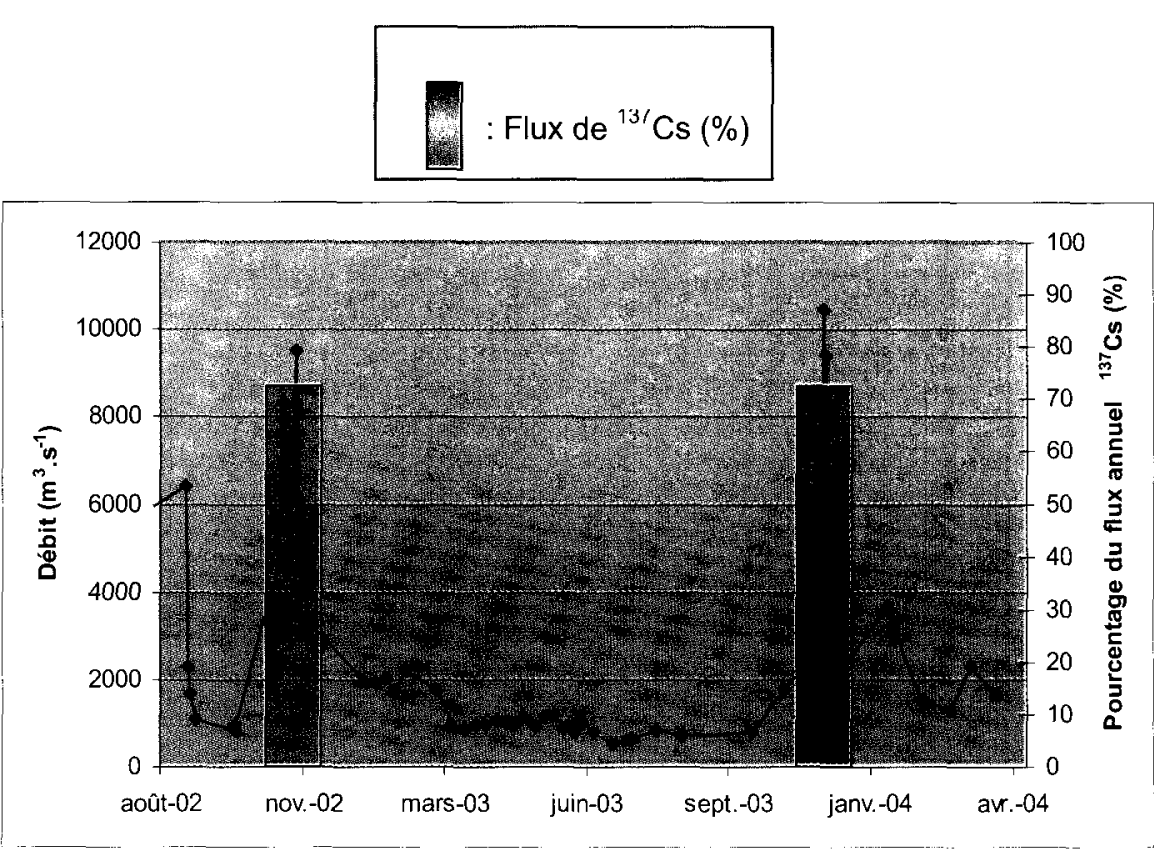

Figure 10.2-5

Évolution du débit liquide du Rhône et importance des deux épisodes de crues rencontrés entre 2002 et 2004 - Flux de ${ }^{137}$ Cs transporté par les deux dernières crues les plus importantes.

Marcoule) mais également des retombées de l'accident de Tchernobyl. En revanche, il n'a pas de rôle biologique connu. De fait, la chronique de cet élément dans des moules prélevées en Méditerranée montre une augmentation des concentrations en 1986 au moment de l'accident de Tchernobyl et reflète assez fidèlement les rejets du centre de retraitement de Marcoule (figure 10.2-6). A noter la réduction drastique des concentrations à partir de 1991 suite à la mise en exploitation d'une nouvelle station de traitement des effluents liquides. À partir de 1999, le ruthénium 106 n'est plus détecté dans les prélèvements en relation avec l'arrêt des opérations de retraitement sur le centre de Marcoule en 1997.

Le césium 137 est également rejeté par les centres de retraitement du combustible mais est aussi un marqueur des retombées des tirs atmosphériques et de l'accident de Tchernobyl, comme le montre la figure 10.2-2. Son évolution temporelle dans les moules à l'embouchure du Rhône (figure 10.2-6) reflète bien les grandes tendances temporelles de ces sources, à savoir une importante réduction dans les rejets de l'usine de Marcoule à partir de 1991 et une augmentation des concentrations suite à l'accident de Tchernobyl. Cependant, l'étude de cet élément à des échelles spatio-temporelles plus fines requiert une 


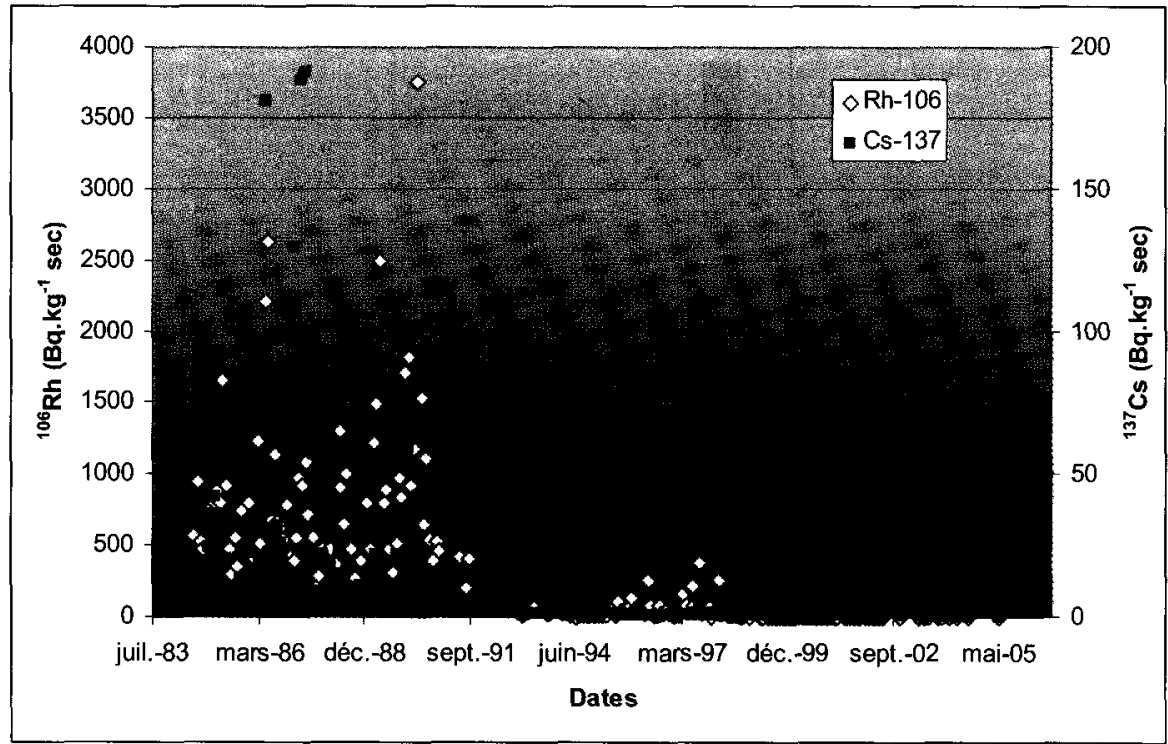

\section{Figure 10.2-6}

Évolutions temporelles des niveaux d'activité massique (Bq. $\mathrm{kg}^{-1}$ frais) du ${ }^{106} \mathrm{Rh}$ et du ${ }^{137} \mathrm{Cs}$ dans les moules prélevées en Méditerranée à la station de Faraman, proche de l'embouchure du Rhône.

bonne connaissance des cycles biologiques des organismes prélevés. En effet, il n'a pas de rôle biologique mais c'est un analogue du potassium dont il mime le comportement et il se fixe donc de manière prépondérante dans les muscles notamment.

Concernant la façade Atlantique, la région de Concarneau représente bien une zone de "référence ", soumise seulement aux radionucléides artificiels des retombées atmosphériques. II en est de même en mer de la Manche Ouest. En Manche centrale et orientale, un marquage par des radionucléides artificiels est observé et est à mettre principalement en relation avec les rejets du centre de retraitement du combustible de la Hague (Nord-Cotentin). Depuis I'entrée en service de l'usine, les rejets en milieu marin ont augmenté, d'une manière générale, jusqu'au début des années 1980, en rapport avec la montée en puissance de l'activité de retraitement. Les procédés de traitement des effluents ont été améliorés, se traduisant par une deuxième phase de très forte diminution des quantités de la plupart des radionucléides rejetés. Les rejets de ruthénium 106, par exemple, ont ainsi baissé d'un facteur 100 depuis les années 1980. Quelques radionucléides échappent cependant à cette évolution des rejets liquides car ils ne sont pas retenus par les procédés de retraitement et sont par conséquent rejetés en proportion du tonnage traité par l'usine. Il s'agit du carbone 14, du tritium et, jusqu'en 1999, de l'iode 129. 
La distribution côtière des radionucléides chez les espèces biologiques est classiquement liée à la distance du point de rejet mais est aussi marquée par les caractéristiques hydrodynamiques particulières de cette région. $D^{\prime}$ un point de vue général, un marquage apparaît autour de la presqu'île du Cotentin (figure 10.2-7), puis les concentrations diminuent, depuis le Cotentin vers l'Est en direction du Pas-de-Calais (figure 10.2-8). La dilution des rejets liquides émis par l'usine Areva NC de La Hague dans la partie orientale de la Manche lest de la presqu'île du Cotentin) se traduit par des concentrations approximativement diminuées de moitié entre le Cotentin et le Pas-de-Calais pour les radionucléides essentiellement solubles.

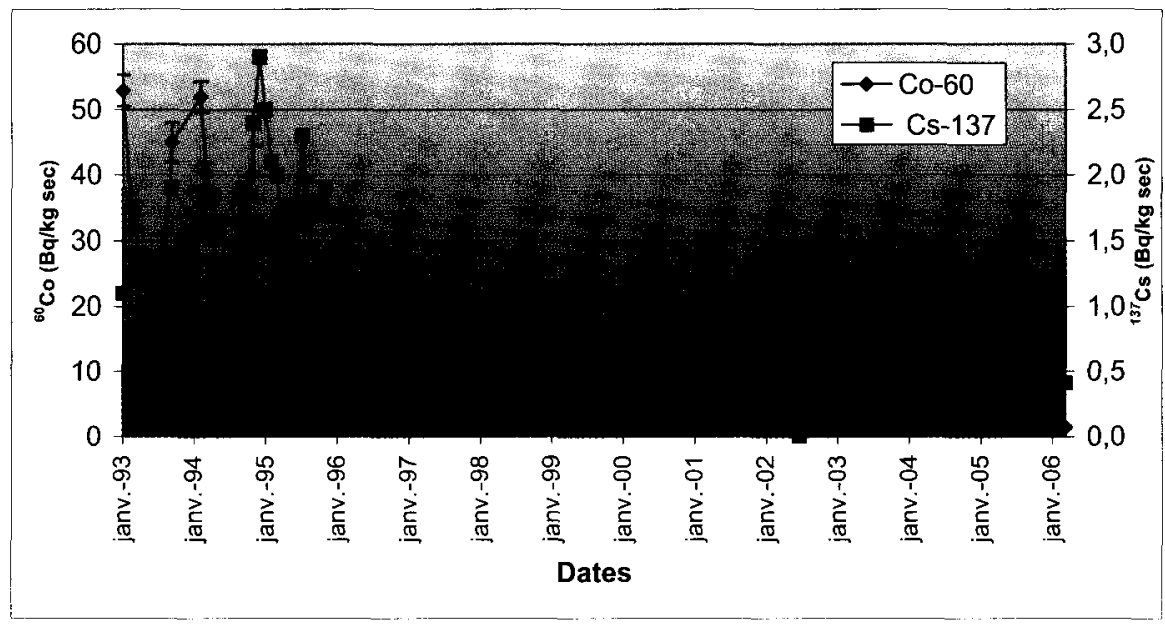

Figure 10.2-7

Évolutions temporelles de l'activité massique du ${ }^{60} \mathrm{Co}$ et du ${ }^{137} \mathrm{Cs}$ dans les algues Fucus Serratus de 1993 à 2006 à Diélette ( $15 \mathrm{~km}$ au sud de l'émissaire d'Areva NC)

Les données de l'observatoire Opera en Manche ont été très utiles pour le Groupe Radioécologie Nord-Cotentin (GRNC, groupe nommé par le gouvernement pour donner un avis sur l'excès de leucémies chez les enfants enregistré dans le Cotentin). Ce groupe a, en particulier, estimé les concentrations en radionucléides dans les différents compartiments du milieu marin du Nord-Cotentin, dans le but de calculer les doses finales reçues par la population locale. La validation de ces estimations n'a pu être réalisée que grâce à leur confrontation avec des mesures de terrain. Les données d'Opera ont été utilisées pour cette action.

Le modèle d'observation Opera marin a été adopté par la CIESM ${ }^{5}$ pour l'organisation d'un réseau international d'étude de la radioactivité dans les moules (Mussel watch) sur l'ensemble du pourtour méditerranéen.

\footnotetext{
${ }^{5}$ Commission internationale pour l'exploration scientifique de la mer Méditerranée.
} 


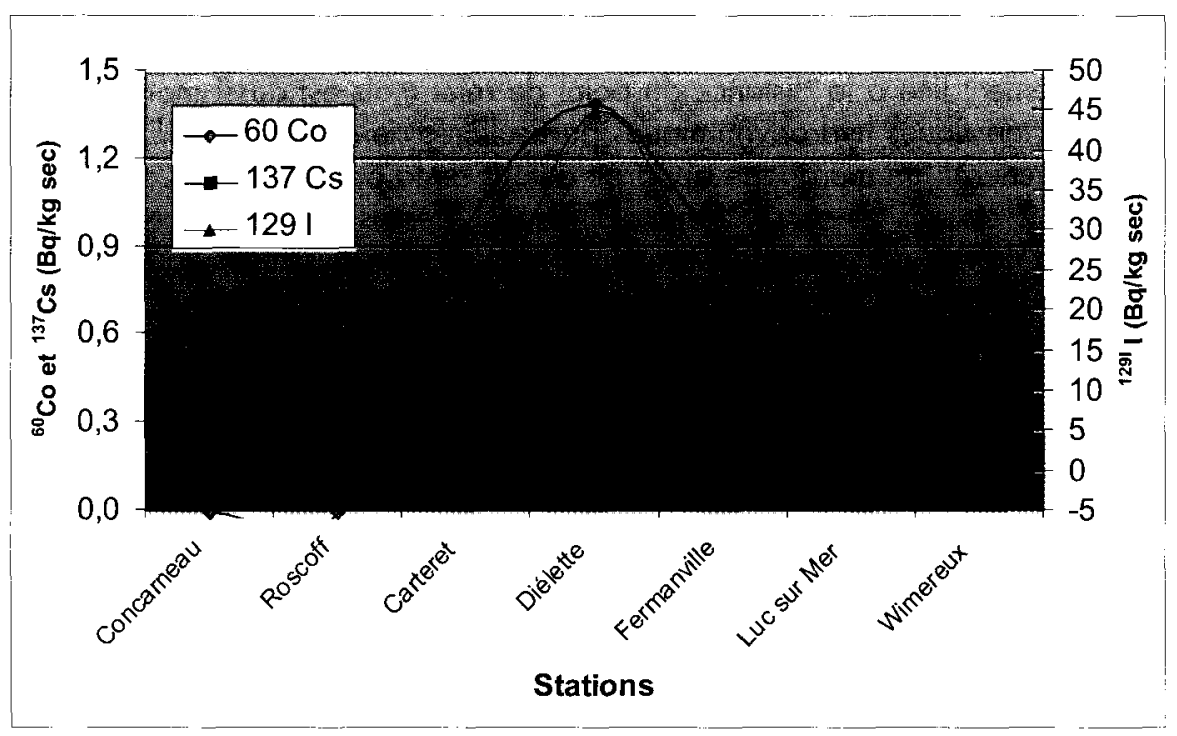

Figure 10.2-8

Évolutions spatiales de l'activité massique du ${ }^{60} \mathrm{Co}, d{ }^{137} \mathrm{Cs}$ et de ${ }^{129} /$ dans les algues Fucus Serratus prélevées en mars 2006 sur les stations Opera de l'Atlantique et de la Manche.

\section{Conclusions et perspectives}

Les observatoires environnementaux ont souvent été considérés comme des outils figés ne s'attachant pas à la connaissance des processus et se bornant plutôt à un constat de l'état du milieu. L'actualité du changement global a apporté une juste reconnaissance de l'apport des observatoires aux sciences de I'Univers.

La nécessaire pérennité d'un observatoire n'empêche pas une évolution régulière des modalités de prélèvement (lieux, échantillons, fréquences), des techniques mises en œuvre en réponse aux questions scientifiques comme aux préoccupations de la société.

Comme cela a été souligné précédemment, des échanges permanents entre observation et recherche sont réalisés dans le cadre d'Opera. Suite à diverses observations, I'IRSN développe des programmes d'études et de recherches, comme le projet Extreme axé sur les processus évènementiels de forte amplitude (pluies torrentielles, poussières désertiques, tempêtes, crues, inondations) qui conduisent à des flux intenses de radionucléides et à une modification de la répartition des stocks de radionucléides artificiels dans les écosystèmes. Ces travaux s'inscrivent dans le cadre plus général des programmes de recherches 
sur le changement global menés par la communauté scientifique. Les progrès de l'instrumentation autonome permettent aujourd'hui d'envisager l'étude de ce type d'évènements qui par essence sont difficiles à échantillonner.

Les études futures doivent mettre l'accent sur le choix de sites clés privilégiant des observations à hautes fréquences et non plus s'appuyer uniquement sur des grandeurs moyennes intégrées dans l'espace ou dans le temps. L'observatoire Opera, dans son évolution, intègre déjà cette complémentarité entre observation à basse fréquence et suivi détaillé à haute fréquence sur plusieurs sites.

Ainsi, le volet atmosphérique s'est enrichi en 2004 d'une station d'étude des niveaux d'activité en altitude avec la mise en service d'une station de collecte d'aérosol et d'eau de nuage à grande capacité, au sommet du Puy-de-Dôme. Comme dans le cas de la plate-forme expérimentale sur le Rhône, d'autres stations multipartenaires et multidisciplinaires sont amenées à se développer dans le cadre des partenariats tissés entre Opera et des stations ou des observatoires des sciences de l'univers (OSU) gérées par des laboratoires de recherches, pour une mise en commun des moyens.

Les retombées de ces dispositifs d'observation dans le domaine opérationnel sont à ce jour encore peu valorisées. Elles sont pourtant essentielles pour les décideurs, notamment en situation post-accidentelle. Cette préoccupation fait actuellement l'objet à l'IRSN d'un projet sur la vulnérabilité des écosystèmes vis-à-vis d'une pollution radioactive (projet Sensib).

Il est important de souligner que la France est l'un des seuls pays au niveau mondial qui anime un observatoire de la radioactivité à l'état de traces dans les différents compartiments de la biosphère.

\section{Références bibliographiques}

Bouisset P., Barker E., Masson O., Gurriaran R., Cagnat X., Mekhlouche D., Aubry S., Hadjaj M. et Saey L. (2004). Concentration de ${ }^{137} \mathrm{Cs}$ et de ${ }^{7} \mathrm{Be}$ dans les aérosols en France de 1959 à 2002. Radioprotection, 39 (3) : 367-381.

UNSCEAR (2000). Sources and effects of ionizing radiation. United Nations Scientific Committee on the Effects of radiation. UNSCEAR 2000 report to the general assembly, with Scientific Annexes. Vol 1 : Sources, United Nations, New York. 
